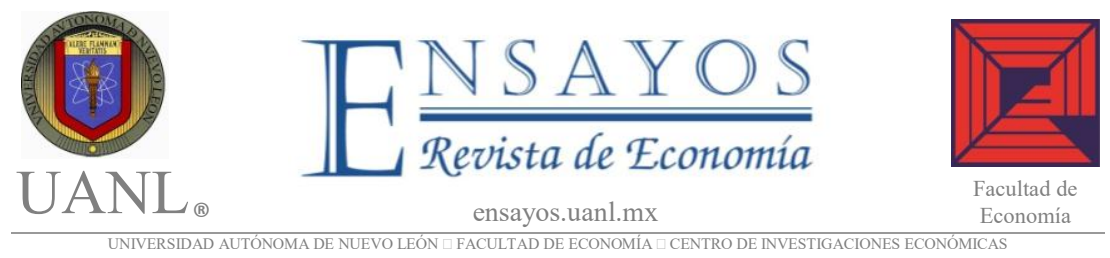

\title{
Salarios, desempleo y productividad laboral en la industria manufacturera mexicana
}

\section{Wage, Unemployment and Labor Productivity in the Mexican Manufacturing Industry}

\section{José Abraham López Machuca* Jorge Eduardo Mendoza Cota}

\begin{tabular}{l}
\hline $\begin{array}{l}\text { Información del } \\
\text { artículo }\end{array}$ \\
\hline Recibido: \\
5 Septiembre 2016 \\
Aceptado: \\
19 Enero 2017 \\
\hline
\end{tabular}

Clasificación JEL: J21; J64; J82; J23

Palabras clave:

Mercado Laboral; Salarios Reales; Curva de Salarios; Desempleo; Productividad Laboral

\section{Resumen}

El artículo evalúa la relación de la productividad laboral y el desempleo con los salarios reales en México durante el periodo 2007 a 2015. Basándose en el modelo de la curva de salarios, la metodología utilizada emplea técnicas econométricas diseñadas para estructuras funcionales estáticas, dinámicas y de cointegración de largo plazo. Los resultados sugieren que los salarios bajos pueden explicarse por la presencia de rigideces importantes, por lo que los salarios guardan una relación inversa con la productividad laboral y el desempleo. Las variaciones en los precios y del salario mínimo no generan cambios significativos en los salarios reales.

\footnotetext{
*Maestro en Economía Aplicada por El Colegio de la Frontera Norte. Correo electrónico: jab_loma@gmail.com

Profesor de tiempo completo del Departamento de Estudios Económicos de El Colegio de la Frontera Norte. Doctor en Economía por la Universidad de Utah, Estados Unidos.

Dirección: Km. 18.5 Carretera Escénica Tijuana - Ensenada, San Antonio del Mar. Tijuana Baja California, México, C. P. 22560. Correo electrónico: emendoza@colef.mx
} 


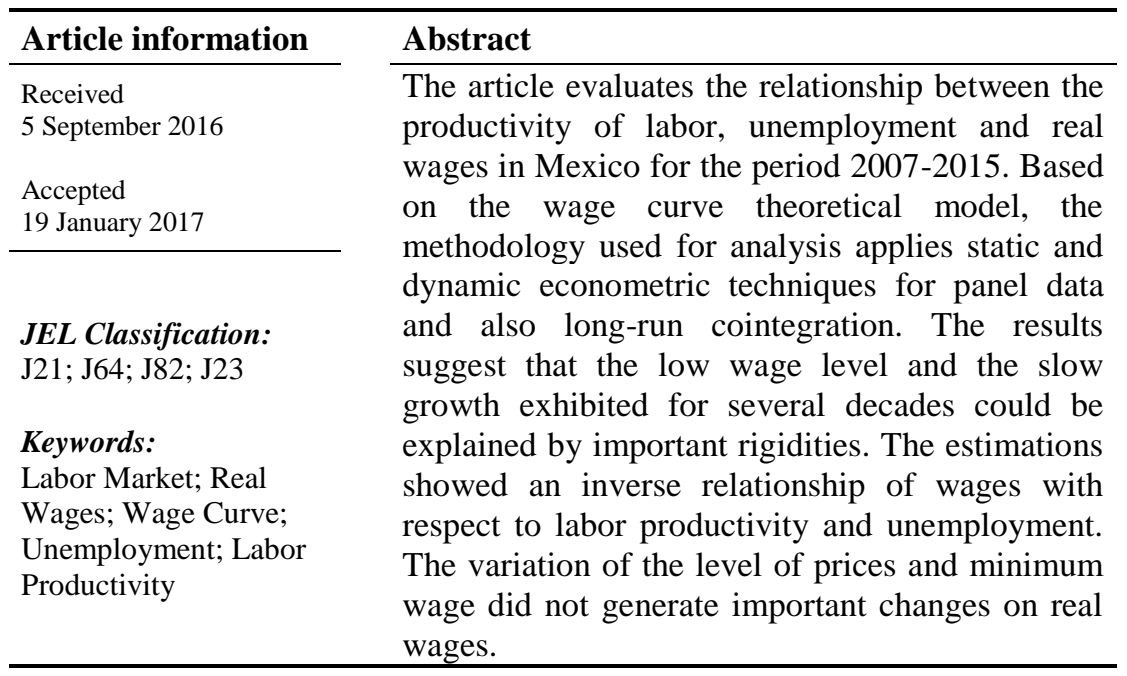

\section{Introducción}

En años recientes, el tema de los salarios en México se ha introducido con mayor fuerza en los debates sostenidos entre integrantes de cámaras legislativas, organizaciones de trabajadores, y con aquellos pertenecientes al sector académico. Esto debido, principalmente, a la considerable reducción en cifras reales de los salarios que carecen incluso de suficiencia en poder adquisitivo para cumplir con las normas constitucionales. Ante esto, el Gobierno presentó a finales de 2012, como respuesta, la reforma más sustancial que se ha realizado a la Ley Federal del Trabajo desde su entrada en vigor en 1970. Y entre sus principales innovaciones está la obligatoria incorporación de los incrementos de la productividad de los trabajadores a partir de los incrementos salariales; es decir, alcanzar la "democratización de la productividad".

Ante lo anterior, y después de tres años de puesta en marcha dicha reforma, resulta importante evaluar la relación que han mantenido los cambios en la productividad laboral con los cambios en los salarios de los trabajadores: Este es el objetivo principal de la investigación, a fin de abrir un panorama que explique el comportamiento futuro de los salarios, bajo este mecanismo.

No obstante, debe recordarse que en el caso del mercado laboral mexicano, se encuentran presentes algunos fenómenos particulares como altos niveles de informalidad laboral y sobretodo la emigración de mano de obra hacia los Estados Unidos, los cuales pueden implicar distorsiones en el ciclo económico de dicho mercado. Adicionalmente, desde el agotamiento del modelo de sustitución de importaciones, la economía mexicana ha mantenido 
como una de sus bases fundamentales la atracción de capitales extranjeros para incidir en la generación de empleos. Dicho mecanismo ha sido factible en gran medida por el sostenido bajo costo de la mano de obra en el mercado internacional, desde hace ya varias décadas y, más aún, sin que esto sea complementado por una política industrial dirigida al fortalecimiento de la estructura económica nacional. De manera conjunta, estos elementos pueden alterar el vínculo entre la productividad y los salarios de los trabajadores, lo que deriva en rigideces dentro de la dinámica salarial e invalida los argumentos de la actual reglamentación laboral.

Para corroborar lo anterior, la investigación se centra en estimar una ecuación de salarios para el caso del sector manufacturero mexicano, asumiendo una perspectiva macroeconómica, en donde la productividad laboral y el nivel de desempleo son los principales determinantes de los ingresos personales de los trabajadores, de acuerdo con lo que establece la teoría económica laboral; pero considerando también otros factores, como las variaciones en el nivel general de precios y el papel del salario mínimo. El estudio se enfoca en el análisis del sector manufacturero debido a su importancia en términos de generación de empleo y contribución en el producto interno bruto. Cabe señalar que, para el sector primario, existe la limitante para cuantificar la productividad laboral tratándose de actividades guiadas por una producción estacionalizada; mientras que para el sector terciario, la actividad está basada en la producción de bienes intangibles. En estos últimos sectores, la cuantificación de la productividad laboral resulta menos factible y una comparación que integre los tres sectores resultaría ambigua.

Por otra parte, se reconoce la importante heterogeneidad que existe en términos temporales y espaciales considerando las contrastantes condiciones económicas, laborales y salariales que existen entre cada una de las 32 entidades federativas del territorio mexicano, así como las diferentes etapas de estabilidad y crisis económica que se han observado en la última década. Con esto, el análisis empírico de este estudio se sustenta metodológicamente por herramientas e información que asume dichas condiciones. En específico, los resultados que se muestran se derivan de modelos econométricos que recogen información tanto de corte transversal como de series de tiempo y que ha sido diseñada y ofrecida por fuentes oficiales especializadas en temas del mercado laboral nacional.

Los argumentos empíricos que se ofrecen en esta investigación asumen, dentro de sus principales resultados, una relación inversa entre los salarios y el desempleo, así como el reconocimiento de un peso importante de elementos institucionales para la determinación de los salarios reales.

El trabajo está organizado de la siguiente forma: en el inciso uno, se hace una revisión de la literatura; en el dos, se presentan los principales planteamientos 
empíricos para la relación productividad y salarios; en el tres, se muestra un panorama contextual para dichas variables en el nivel regional y estatal; en el cuatro, se expone la estrategia metodológica a seguir, así como los resultados obtenidos. Finalmente, se presentan las principales conclusiones de la investigación.

\section{Elementos teóricos con fundamentos microeconómicos}

Dentro de la literatura referente a determinantes de salarios, es posible encontrar planteamientos teóricos que hablan sobre el papel del capital humano (la educación, las formas de capacitación laboral y la experiencia profesional de los individuos), el capital económico (la infraestructura para los medios de producción) y los rasgos individuales de los trabajadores (edad, sexo, estado civil, entre otros). En conjunto, dichos planteamientos analizan la dinámica de los salarios y dan explicación a fenómenos como el desempleo involuntario y las diferencias salariales, basándose en mercados con información incompleta entre los agentes y en los mecanismos de negociación salarial y contratación, siendo estos los principales elementos que generan rigideces salariales en mercados imperfectos.

Asimismo, la aceptación de diferentes niveles de salarios con respecto al nivel general del mercado ha derivado en el concepto de salario de eficiencia, el cual puede ser entendido como un salario superior al salario promedio del mercado, mediante el cual se minimizan costos derivados del proceso de contratación laboral y promueve una mayor productividad para los trabajadores. Dentro de este esquema, se encuentran los modelos que a continuación se citan.

El modelo de shirking de Shapiro y Stiglitz (1984) cuestiona el que los salarios no ajusten la ocupación hacia su nivel de equilibrio, como lo dicta la teoría marginalista. Los autores muestran que la estructura de la información en el mercado laboral explica el desempleo involuntario, dada la incapacidad de los empleadores para observar el esfuerzo de los trabajadores, sin incurrir en un costo. En este contexto, los empleadores no tienen un monitoreo perfecto del desempeño de los trabajadores. El ofrecimiento de un salario de eficiencia garantiza que los trabajadores realicen su máximo esfuerzo minimizando el costo del empresario por unidad efectiva empleada. Al pagar un salario superior al del mercado, si el trabajador es sorprendido en un bajo rendimiento, será despedido y solo podrá ser empleado en otro lugar con un salario menor. Este modelo representa el modelo básico de salarios de eficiencia; según Akerlof (1980) y Akerlof y Yellen (1990), el salario de eficiencia debe considerarse como un elemento de justicia hacia los trabajadores, quienes evalúan el trato de la empresa en función del nivel de salario concedido. 
Por otra parte, Azariadis y Stiglitz (1983) y Stiglitz (1986) desarrollaron el modelo de contratos implícitos, el cual destaca que los trabajadores no pueden protegerse contra cambios en sus ingresos a través de compañías convencionales de seguros, y tienen un acceso limitado al mercado de crédito. Contrariamente, los empleadores resultan menos adversos al riesgo y tienen un mayor acceso al mercado de capitales. Como resultado, los empleadores pueden ofrecer algún tipo de seguro a sus trabajadores, como parte del contrato de trabajo, para atraer mano de obra con un nivel de salario menor, siendo este seguro una prestación adicional que asume el papel de salario de eficiencia.

El modelo de histéresis o enfoque insider-outsider, desarrollado por Lindbeck y Snower (1986), Blanchard y Summers (1987) y Gregory (1986), critica el supuesto neoclásico de libre entrada y salida del mercado de trabajo. El modelo analiza las causas de la fijación de los salarios en un nivel por encima al de equilibrio, y otorga cierto poder de mercado a los trabajadores para la fijación del nivel de salario. El fundamento principal de esta teoría es que la rotación del personal en una empresa, a través de un intercambio entre los trabajadores ocupados (insiders) y los trabajadores que se encuentran desocupados (outsiders), resulta costosa para la empresa, si se consideran los costos de capacitación, de despido y de contratación. Esto permite que los insiders eleven sus salarios por encima del salario de equilibrio, sin que sea posible su sustitución por un trabajador outsider.

Finalmente, el modelo de matching asume elecciones individuales basadas en información completa que permiten que los trabajadores transiten de un trabajo a otro de forma inmediata, sin que experimenten periodos de búsqueda de empleo. Sin embargo, desde finales del decenio de 1960, se observó que la duración del desempleo se incrementa en periodos contracíclicos. Dicha duración se explica subrayando que los trabajadores desocupados analizan las opciones de inserción en el mercado de trabajo considerando diversos factores además del salario. Como lo menciona Mortensen (1986), reconociendo que la información respecto de las vacantes y los salarios ofrecidos no es perfecta e implica un costo para ambas partes (empleador y empleado), el salario percibido, una vez empleado, dependerá de los costos implícitos en dicha búsqueda. Las implicaciones de este planteamiento se relacionan con el hecho de que los trabajadores deberán definir una estrategia para determinar cuándo el salario que les es ofrecido es aceptable. Lo anterior implica que la asimetría y los costos derivados desvinculan la oferta de empleo y los salarios de los trabajadores, fenómeno que ha sido verificado empíricamente. 


\section{Estudios empíricos}

El análisis empírico de la determinación de salarios en el nivel macroeconómico parte de la relación entre salarios y desempleo con el modelo de la curva de Phillips y el modelo de Harris-Todaro. Phillips (1958), utilizando datos anuales para el caso del Reino Unido, encontró que la relación entre las tasas de cambio de los salarios monetarios y el desempleo mostraba una curva con pendiente negativa. Friedman (1968) y Phelps (1968), asumiendo información incompleta entre los agentes económicos y una tasa natural de desempleo, diferenciaron los efectos transitorios de corto y largo plazo entre inflación y desempleo.

Harris y Todaro (1970) plantearon una visión alternativa en cuanto a la relación salarios-desempleo, desarrollando un modelo compuesto por un sector urbano y un sector rural, donde existen salarios urbanos que en su nivel mínimo son sustancialmente mayores a los salarios en la agricultura. Adicionalmente, diversos estudios basados en esta visión han explicado las diferencias salariales entre regiones, destacando a Hall, Gordon and Holt (1972), Reza (1978), Roback (1982), Marston (1985), Adams (1985) y Topel (1986).

En la década de los ochenta, se realizaron diversos estudios que mostraron una relación en sentido opuesto y que sería definida, por Blanchflower y Oswald (1990), como la curva de salarios. La relación negativa entre salarios y desempleo converge con los planteamientos realizados por la curva de Phillips, sin embargo existen tres diferencias importantes. Primero, la curva de Phillips vincula la tasa de cambio de los salarios con la tasa de desempleo, mientras que la curva de salarios vincula los salarios en niveles con la tasa de desempleo; segundo, la curva de Phillips se relaciona con la inflación, la curva de salarios no; y tercero, la curva de Phillips trata acerca de los efectos del desempleo agregado, en cambio la curva de salarios, del papel del desempleo local. Asimismo, se puede establecer que la curva de Phillips establece un esquema macroeconómico utilizando la variable agregada de desempleo como mecanismo de ajuste; la curva de salarios refiere a un equilibrio que se mantiene en el tiempo y que es ajeno a dinámicas transitorias, explicado a partir de variables microeconómicas o individuales. Como antecedentes más relevantes de la curva de salarios, se pueden mencionar los trabajos de Blackaby y Manning (1987), Layard y Nickell (1986), Freeman (1988), Pissarides y McMaster (1990), Blanchard y Katz (1992) y Jones (1989). De manera general, en dichas investigaciones se converge sobre la existencia de un efecto negativo en los salarios en el desempleo. 
Blanchflower y Oswald (1994) presentaron estudios realizados para los casos de Gran Bretaña, Alemania, Australia, Italia, Holanda, Irlanda, Suecia, Noruega, Canadá, Corea del Sur y Australia. En cada caso, se utilizaron datos tipo panel que en conjunto suman información para cerca de 3 millones y medio de personas, con lo que los autores aplicaron el método de mínimos cuadrados generalizados (MCO) a una ecuación en donde interviene el logaritmo de los salarios reales como variable por explicar, a partir del logaritmo del desempleo y un conjunto de variables de control para el tiempo, el espacio y las características particulares de los trabajadores (edad, sexo, escolaridad, raza, sindicalización o sector en el que laboran). Sus estimaciones arrojan una elasticidad del salario ante el desempleo regional de -0.1. Los autores autodenominaron a la curva de salarios: regla empírica de la economía, asumiendo que el comportamiento persiste sin importar el tiempo, la región o el contexto institucional del que se hable. La curva de salarios se respaldó en los modelos cuya base principal se relacionaba con el concepto de salarios de eficiencia, descrito anteriormente.

Para el caso de México, Castro (2006) utilizó información de la Encuesta Nacional de Empleo Urbano (ENEU) y construyó una base de datos para trabajadores de 33 centros urbanos, para el periodo que va de 1993 a 2002. Estimando un modelo de panel dinámico, se evalúa la elasticidad de los salarios ante el desempleo con rezago de un periodo, incluyendo variables dicotómicas para diferenciar las características de los trabajadores, la región en la que laboran, así como el tiempo. Los resultados muestran una elasticidad de -0.03 , siendo esta menor a lo mostrado en el trabajo original de Blanchflower y Oswald (1994).

Partiendo de este esquema microeconómico de la curva de salarios, en la última década, se han realizado investigaciones con una perspectiva mayormente macroeconómica, para retomar la relación inversa entre los salarios y el desempleo, y además incluir la productividad de los trabajadores. Lo anterior, derivado en gran medida de las recomendaciones de organismos internacionales del sector laboral, como la Organización Internacional del Trabajo (OIT), cuyo principal pronunciamiento para sacar a los salarios del estancamiento ha sido a través de la incorporación de los incrementos de la productividad de los trabajadores en sus salarios. Argumento recogido por la última reforma laboral, en el caso mexicano.

Al respecto, Wakeford (2004) analiza el caso de Sudáfrica para el periodo de 1983 a 2002. Utilizando el análisis de cointegración multivariable de Johansen y la prueba de causalidad de Granger, él encuentra la existencia de una relación de equilibrio de largo plazo entre los salarios y la productividad laboral, en donde por cada incremento del $1 \%$ en la productividad, los salarios tendrían un incremento de $0.58 \%$. No obstante, el autor no encuentra 
evidencia de una relación de equilibrio de largo plazo entre el desempleo y el resto de las variables.

Goh y Wong (2010) emplean técnicas de corrección de error y cointegración multivariable para datos de Malasia entre 1970 y 2005. En el estudio, se encuentra una relación positiva de equilibrio de largo plazo entre los salarios y la productividad, pero no así para los salarios con el desempleo. Otros trabajos, utilizando técnicas de cointegración, encontraron que la elasticidad de los salarios con respecto a la productividad fue mayor a la unidad, como el caso de Israel, documentado por Lavy y Sussman (2001), Australia (Kumar, et al., 2012), Reino Unido (Alexander, 1993) y Estados Unidos, analizado por Strauss y Wohar (2004). Dichos resultados sugieren que, en el largo plazo, la productividad ejerce un efecto significativo sobre los salarios, y que estos últimos tienden a desasociarse de los niveles de desempleo.

Para el caso de México, Castellanos (2010) evalúa la relación entre salarios nominales, desempleo y productividad laboral al interior del sector manufacturero, para el periodo de 1994 a 2001. Mediante el Método Generalizado de Momentos (MGM) para datos panel dinámicos, se concluye que el desempleo modera el incremento de los salarios, mientras que la productividad lo incrementa, entre -0.5 y 0.25 unidades, respectivamente. Galindo y Catalán (2010) evalúan la hipótesis de la curva de salarios con información trimestral para el periodo de 1989 a 2008. Utilizando el análisis de cointegración e incluyendo a la productividad laboral como variable explicativa, además de una variable de precios y un salario de reserva medido a través del salario mínimo, los autores obtuvieron una elasticidad salariosdesempleo de -0.032; mientras que la productividad, la escala de precios y el salario mínimo impactan de manera positiva en un nivel de $0.27,0.82$ y 0.07 , para cada caso.

A manera de síntesis, dentro de los planteamientos relacionados con los determinantes salariales, los elementos teóricos microeconómicos relacionados con el concepto de salario de eficiencia han sido corroborados a través de modelos empíricos, como la curva de salarios, para posteriormente integrarse a modelos macroeconómicos que permiten la inclusión de la productividad laboral, misma que había sido excluida asumiendo una mayor importancia por parte del desempleo y la inflación.

\section{Evolución del mercado laboral del sector manufacturero}

Este apartado es destinado a describir la dinámica de las principales variables analizadas en la presente investigación como lo son: producción, remuneraciones y productividad laboral, para contextualizar el caso del sector manufacturero mexicano, desagregando su desarrollo en el nivel de cada una 
de las 32 entidades, así como las ramas y subsectores que componen dicho sector. A su vez, esta información permitirá entender el comportamiento de las variables en su posterior uso dentro del análisis econométrico sugerido.

A partir de la década de 1980 y, en particular, con la formalización del Tratado de Libre Comercio de América del Norte (TLCAN), es posible observar cambios importantes en la estructura productiva de las manufacturas mexicanas. Por una parte, la producción de las industrias tradicionales ha venido declinando en favor de la producción de industrias más intensivas en capital y conocimiento tecnológico, orientadas hacia la exportación (Rivera, 2001); por otra, la liberalización comercial ha generado un proceso de descentralización de la actividad manufacturera de los estados del centro de México hacia otras estados en el norte del país.

De acuerdo con el Sistema de Clasificación Industrial de América del Norte (SCIAN), al interior del sector manufacturero, es posible identificar tres subsectores cuyas dinámicas han operado en favor de ramas con mayor uso de capital. El subsector 33, que incluye una alta participación por parte de la industria automotriz (fabricación de equipo de transporte), incrementó su tasa de participación en el PIB manufacturero entre 1995 y 2015, pasando de un $33.2 \%$ a un $38.1 \%$ (cuadro 1). Asimismo, dicho subsector ha logrado superar al subsector 31, que fue el más importante en la década de 1990 y que incluye a las industrias más tradicionales como la alimentaria y textil, y al subsector 32 , compuesto por industrias que dependen de la disponibilidad de los recursos naturales, como la maderera y la petrolera ${ }^{\mathbf{1}}$.

En términos del empleo, la industria manufacturera absorbe cerca del $16 \%$ de la población ocupada en México y, en la última década, el dinamismo del empleo manufacturero se ha mantenido similar al comportamiento de la mayoría de los sectores, con tasas medias de crecimiento anual cercanas al $1.5 \%$ cada año (cuadro 2 ).

El sector automotriz absorbió en 2015 el $22.3 \%$ de los trabajadores del sector manufacturero, acercándose al $30.5 \%$ del total de las industrias del subsector 31, que muestra disminuciones en los últimos años. Esto se puede observar tomando el número de empleos generados, como saldo neto para este periodo, en el sector automotriz, que fue de 238,434 plazas laborales. De hecho, el subsector 33 fue el único en registrar cifras netas positivas en generación de empleo (cuadro 3).

\footnotetext{
${ }^{1}$ Estimaciones propias con datos del Sistema de Cuentas Nacionales de México del INEGI.
} 


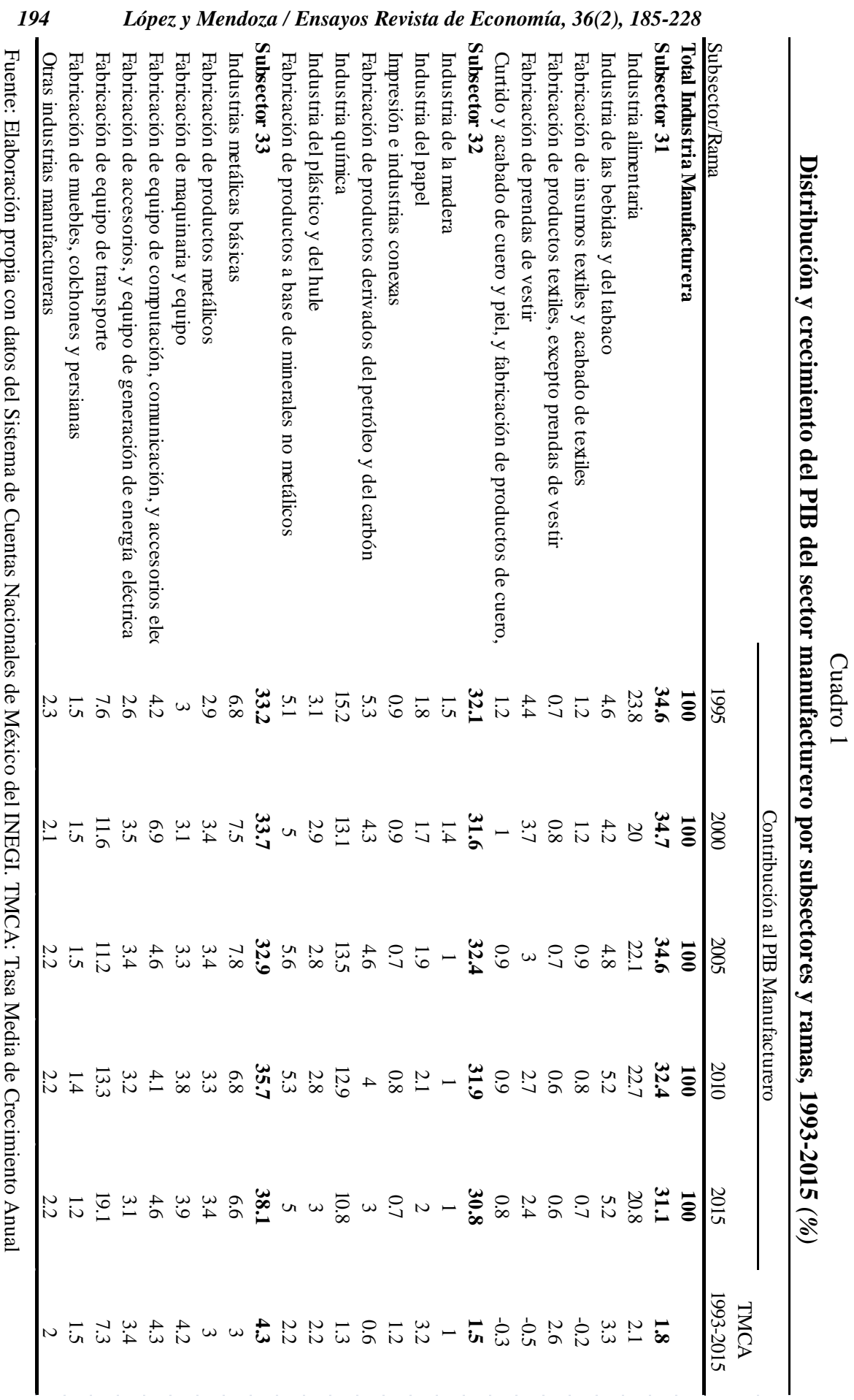


Cuadro 2

Distribución y crecimiento sectorial del empleo en México, 2005-2015

\begin{tabular}{|c|c|c|c|}
\hline \multirow[b]{2}{*}{ Sector } & \multicolumn{2}{|c|}{ Participación } & \multirow{2}{*}{$\begin{array}{c}T M C A \\
2006-2015\end{array}$} \\
\hline & 2005 & 2015 & \\
\hline Total Actividad Económica & 100 & 100 & \\
\hline $\begin{array}{l}\text { Agricultura, ganadería, } \\
\text { silvicultura, caza y pesca }\end{array}$ & 14.8 & 13.3 & 0.8 \\
\hline $\begin{array}{l}\text { Industria extractiva y de } \\
\text { la electricidad }\end{array}$ & 0.9 & 0.8 & 1.8 \\
\hline Industria manufacturera & 16.8 & 16 & 1.5 \\
\hline Construcción & 7.9 & 7.8 & 1.8 \\
\hline Comercio & 19.6 & 19.2 & 1.7 \\
\hline $\begin{array}{l}\text { Restaurantes y servicios } \\
\text { de alojamiento }\end{array}$ & 5.9 & 7.2 & 4 \\
\hline $\begin{array}{l}\text { Transportes, } \\
\text { comunicaciones, correo y } \\
\text { almacenamiento }\end{array}$ & 5 & 4.9 & 1.7 \\
\hline $\begin{array}{l}\text { Servicios profesionales, } \\
\text { financieros y corporativos }\end{array}$ & 5.4 & 7 & 4.6 \\
\hline Servicios sociales & 8.2 & 8.1 & 1.7 \\
\hline Servicios diversos & 10.1 & 10.6 & 2.4 \\
\hline $\begin{array}{l}\text { Gobierno y organismos } \\
\text { internacionales }\end{array}$ & 4.7 & 4.5 & 1.3 \\
\hline No especificado & 0.7 & 0.6 & -0.2 \\
\hline
\end{tabular}

Fuente: Elaboración propia con datos de la ENOE. Indicadores estratégicos del INEGI, México. TMCA: Tasa Media de Crecimiento Anual

La productividad laboral - estimada como el cociente entre la producción y el empleo, a partir de información de la Encuesta Mensual de la Industria Manufacturera (EMIM) - muestra que al interior de la industria manufacturera y de manera individual, la tasa para el total de la industria fue de tan solo $0.7 \%$, entre 2008 y 2015; si bien, en ramas como la industria maderera, de transporte y textil se registró una Tasa Media de Crecimiento Anual (TMCA) mayor (gráfica 1). 


\section{Cuadro 3}

Empleos generados en el sector manufacturero y su distribución, 2007-2015

\begin{tabular}{|c|c|c|c|c|c|}
\hline \multirow{2}{*}{ Rama } & \multicolumn{2}{|c|}{$(\%)$} & \multicolumn{3}{|c|}{ Empleos generados } \\
\hline & 2007 & 2015 & 2007-2009 & 2010-2015 & 2007-2015 \\
\hline $\begin{array}{l}\text { Total Industria } \\
\text { Manufacturera }\end{array}$ & 100 & 100 & $-208,022$ & 406,009 & 197,987 \\
\hline Subs ector 31 & 34.1 & 30.5 & $-60,757$ & 5,902 & $-54,855$ \\
\hline Industria alimentaria & 19 & 18.8 & $-7,978$ & 24,234 & 16,256 \\
\hline $\begin{array}{l}\text { Industria de las bebidas y } \\
\text { del tabaco }\end{array}$ & 4.2 & 3.5 & $-3,586$ & $-5,283$ & $-8,868$ \\
\hline $\begin{array}{l}\text { Fabricación de insumos } \\
\text { textiles y acabado de } \\
\text { textiles }\end{array}$ & 2.2 & 1.6 & $-13,818$ & $-2,126$ & $-15,943$ \\
\hline $\begin{array}{l}\text { Fabricación de productos } \\
\text { textiles, excepto prendas de } \\
\text { vestir }\end{array}$ & 0.7 & 0.6 & $-3,797$ & 1,147 & $-2,650$ \\
\hline $\begin{array}{l}\text { Fabricación de prendas de } \\
\text { vestir }\end{array}$ & 6 & 4.2 & $-24,766$ & $-14,287$ & $-39,053$ \\
\hline $\begin{array}{l}\text { Curtido y acabado de } \\
\text { cuero, piel y materiales } \\
\text { sucedáneos }\end{array}$ & 2 & 1.9 & $-6,812$ & 2,216 & $-4,596$ \\
\hline Subs ector 32 & 17.8 & 16.9 & $-21,582$ & 16,524 & $-5,059$ \\
\hline Industria de la madera & 0.6 & 0.4 & $-2,319$ & $-1,552$ & $-3,871$ \\
\hline Industria del papel & 2.1 & 2 & -406 & 1,058 & 652 \\
\hline $\begin{array}{l}\text { Impresión e industrias } \\
\text { conexas }\end{array}$ & 1 & 0.9 & $-1,278$ & $-2,282$ & $-3,560$ \\
\hline $\begin{array}{l}\text { Fabricación de productos } \\
\text { derivados del petróleo y del } \\
\text { carbón }\end{array}$ & 0.9 & 0.8 & 67 & -869 & -802 \\
\hline Industria química & 4.9 & 4.3 & $-3,398$ & $-5,259$ & $-8,657$ \\
\hline $\begin{array}{l}\text { Industria del plástico y del } \\
\text { hule }\end{array}$ & 5.3 & 5.6 & $-10,271$ & 21,912 & 11,641 \\
\hline $\begin{array}{l}\text { Fabricación de productos a } \\
\text { base de minerales no } \\
\text { metálicos }\end{array}$ & 3.1 & 2.8 & $-3,978$ & 3,516 & -462 \\
\hline Subsector 33 & 48.1 & 52.6 & $-125,683$ & 383,583 & 257,901 \\
\hline $\begin{array}{l}\text { Industrias metálicas } \\
\text { básicas }\end{array}$ & 2.3 & 2.5 & 258 & 11,793 & 12,051 \\
\hline $\begin{array}{l}\text { Fabricación de productos } \\
\text { metálicos }\end{array}$ & 7.1 & 6.6 & 8,636 & $-2,551$ & 6,084 \\
\hline $\begin{array}{l}\text { Fabricación de maquinaria } \\
\text { y equipo }\end{array}$ & 2.6 & 3 & $-4,997$ & 20,734 & 15,736 \\
\hline $\begin{array}{l}\text { Fabricación de equipo y } \\
\text { accesorios electrónicos }\end{array}$ & 8.9 & 7.5 & $-35,835$ & 9,517 & $-26,318$ \\
\hline $\begin{array}{l}\text { Fabricación de aparatos } \\
\text { eléctricos }\end{array}$ & 5.6 & 4.9 & $-18,643$ & 9,376 & $-9,267$ \\
\hline $\begin{array}{l}\text { Fabricación de equipo de } \\
\text { transporte }\end{array}$ & 15.9 & 22.3 & $-68,778$ & 307,212 & 238,434 \\
\hline $\begin{array}{l}\text { Fabricación de muebles, } \\
\text { colchones y persianas }\end{array}$ & 1.7 & 1.4 & $-7,708$ & 1,870 & $-5,838$ \\
\hline $\begin{array}{l}\text { Otras industrias } \\
\text { manufactureras }\end{array}$ & 4 & 4.5 & 1,385 & 25,634 & 27,018 \\
\hline
\end{tabular}

Fuente: Elaboración propia con datos de la Encuesta Mensual de la Industria Manufacturera (EMIM) del INEGI. 


\section{Gráfica 1}

Tasa media de crecimiento anual de la productividad laboral por rama del sector manufacturero, 2008-2015 (\%)

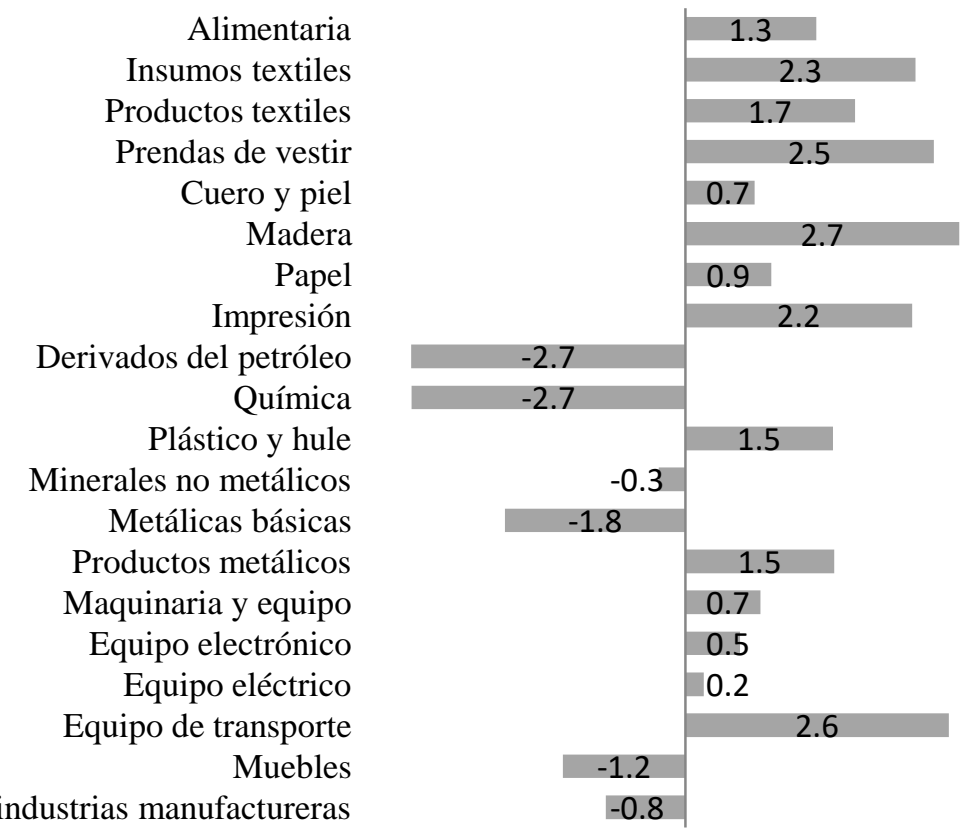

Otras industrias manufactureras

Fuente: Elaboración propia con datos de la Encuesta Mensual de la Industria Manufacturera (EMIM) del INEGI.

Debe destacarse que la productividad laboral del sector manufacturero ha sido influenciada más por cambios en la tendencia de la producción que por cambios en el empleo. La caída o relativa estabilidad del empleo en términos absolutos explica el aumento de la productividad del trabajo, tal y como se mostró para décadas anteriores (CEFP, 2004). En las ramas vinculadas con la industria textil, la industria de la madera, la industria del vestido y la industria de la piel, el crecimiento de la productividad puede ser explicado únicamente por disminuciones en el personal ocupado (gráfica 2). En la rama automotriz, la de maquinaria y equipo y la alimentaria, el crecimiento en la productividad se presentó con un saldo positivo tanto en el empleo como en producción, infiriendo que el crecimiento de la mayor eficiencia productiva fue mayor al crecimiento del empleo (gráfica 2 y 3 ) 

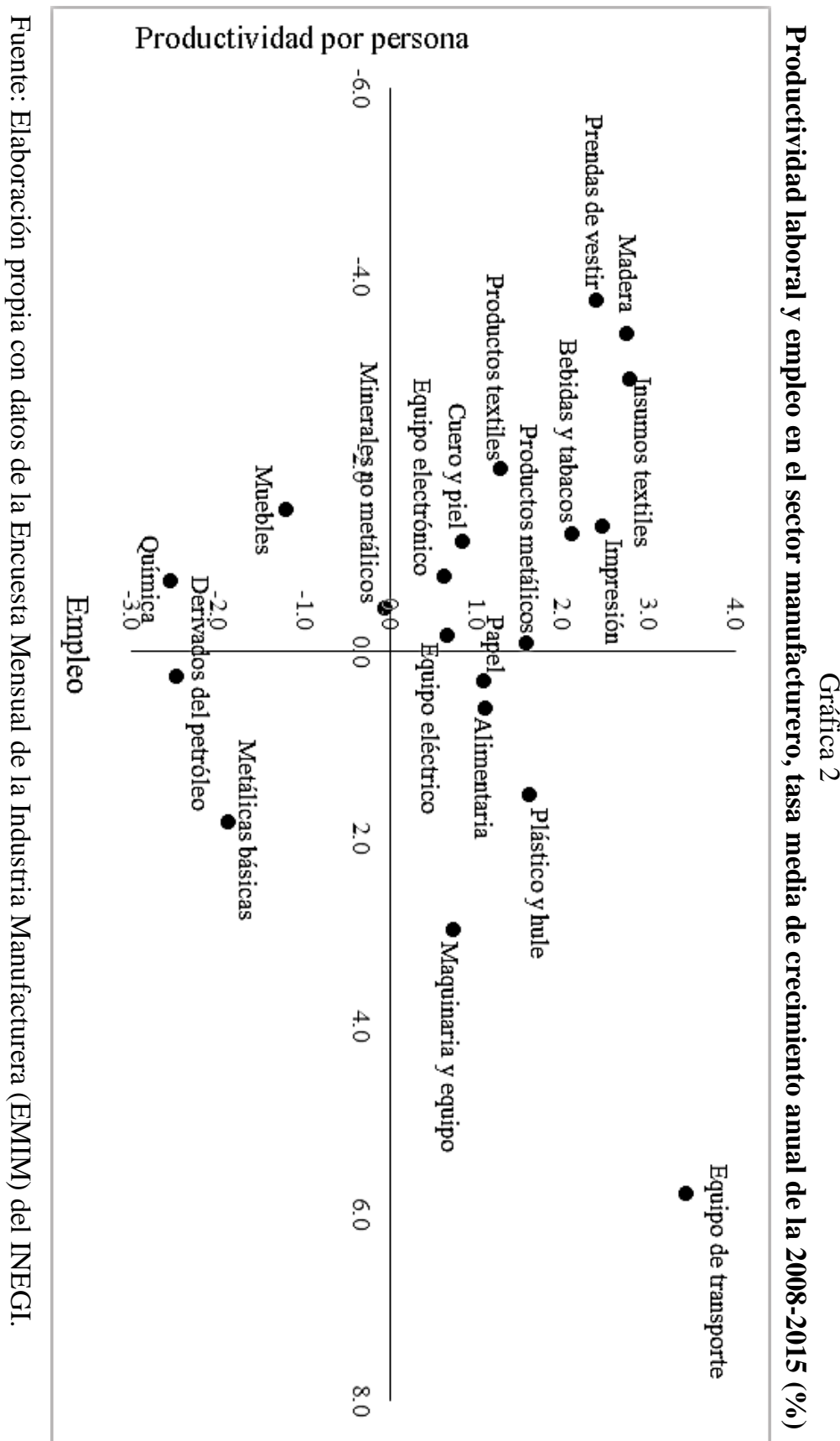


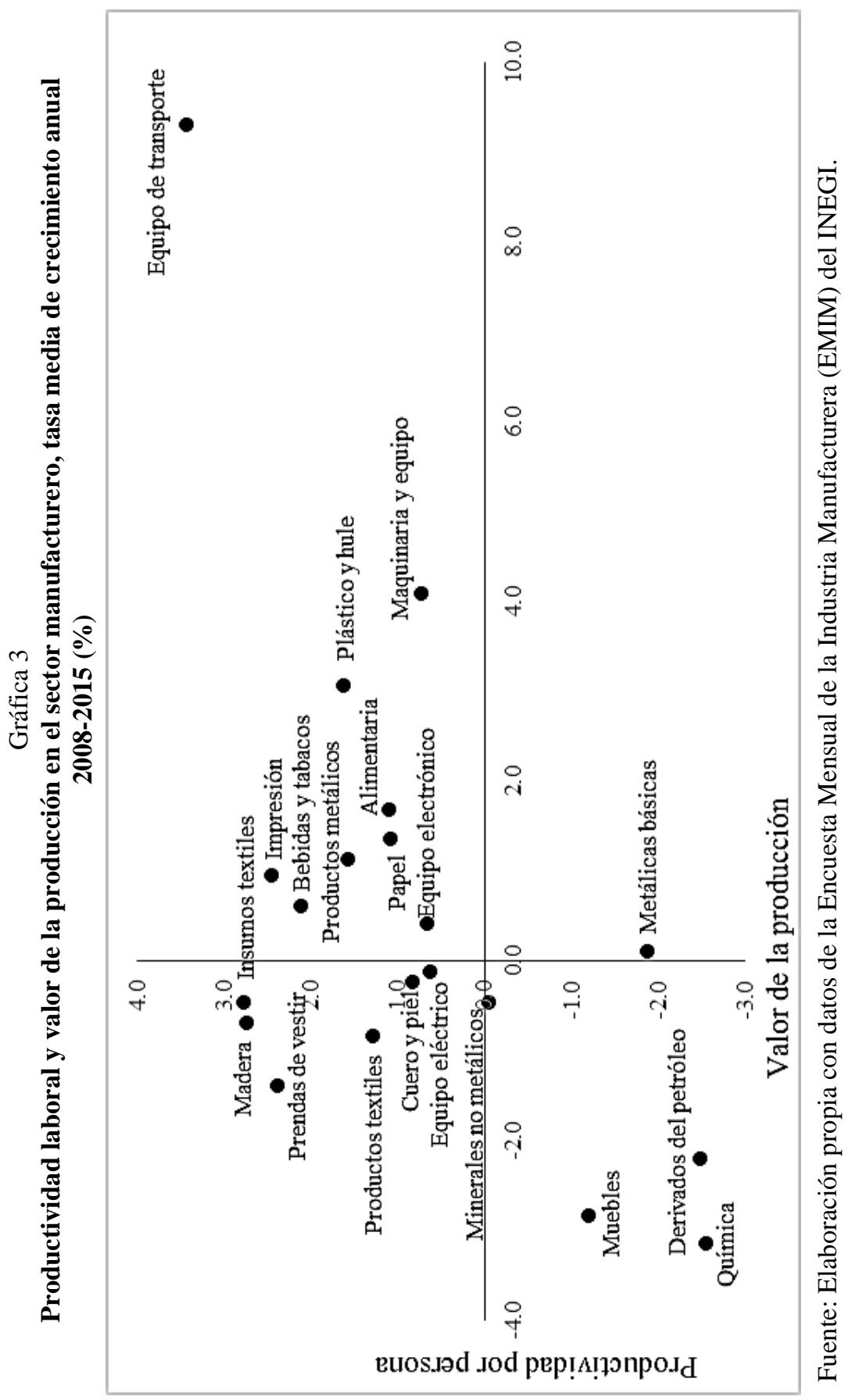


No obstante, aunque la productividad de la fuerza laboral en la industria manufacturera ha sido mínimo a lo largo de la última década, el nivel de remuneración presenta retrocesos mostrando una TMCA de $-1.5 \%$, con reducciones anuales importantes hasta 2012, con una recuperación de $2.1 \%$ en años subsecuentes, pero sin alcanzar el mismo nivel de 2007.

De forma más clara, como se observa en el cuadro 4, en 2011 las remuneraciones mensuales por trabajador tuvieron una pérdida de 1,600 pesos con respecto a lo percibido en 2007; mientras que en 2015 , se observa una ganancia de tan solo 600 pesos, el saldo neto fue de una reducción de mil pesos mensuales en las remuneraciones que percibió un trabajador promedio del sector manufacturero.

En particular, las industrias relacionadas con la fabricación de consumo final, como la alimentaria, de las bebidas y del tabaco, la textil, así como la automotriz, fueron las ramas con mayores reducciones en términos de remuneraciones, debido a los efectos de la crisis, lo cual se observa en las cifras entre 2007 y 2012.

Es posible establecer que, una vez terminada la crisis en el sector manufacturero, las remuneraciones no se ajustan en igual magnitud que la producción, y que la estructura salarial, a la cual se han adaptado las empresas durante periodos de crisis, permanece en niveles inferiores, incluso una vez superada dicha crisis. Este comportamiento puede observarse comparando el crecimiento de las remuneraciones y el de la productividad, como lo muestra gráfica 4. Para el periodo que va del término de 2009 hasta finales de 2012, el crecimiento de la productividad y las remuneraciones de los trabajadores en el sector manufacturero comparten una tendencia similar dentro de las variaciones mostradas, sin embargo, para el caso de las remuneraciones, los incrementos tienen una magnitud menor.

Así mismo, la apertura comercial promovió un proceso de desconcentración de la actividad manufacturera que orientaría a las empresas manufactureras a establecerse en otras regiones, como la zona de la frontera norte del país, principalmente. De esta manera, la Ciudad de México ha tendido a especializarse en actividades terciarias, mientras que Guadalajara y Monterrey continuaron con el desarrollo industrial, adaptándose a las transformaciones que surgían de la apertura comercial. 


\section{Cuadro 4}

\section{Remuneraciones medias mensuales por periodo en el sector manufacturero según rama, 2007-2015 (Miles de pesos)}

\begin{tabular}{|c|c|c|c|c|c|c|c|c|c|}
\hline Rama & 2007 & 2008 & 2009 & 2010 & 2011 & 2012 & 2013 & 2014 & 2015 \\
\hline $\begin{array}{l}\text { Total Industrias } \\
\text { manufactureras }\end{array}$ & 11.3 & 10.6 & 10.6 & 10.1 & 9.7 & 9.4 & 9.6 & 9.8 & 10 \\
\hline Subsector 31 & 8.6 & 7.6 & 7.4 & 7.1 & 6.9 & 6.8 & 7 & 7.1 & 7.2 \\
\hline Industria alimentaria & 8.5 & 7.5 & 7.4 & 7 & 6.8 & 6.7 & 7 & 7.1 & 7.1 \\
\hline $\begin{array}{l}\text { Industria de las bebidas } \\
\text { y del tabaco }\end{array}$ & 12.8 & 10.3 & 9.9 & 9.6 & 9.3 & 8.9 & 8.9 & 8.3 & 8.5 \\
\hline $\begin{array}{l}\text { Fabricación de insumos } \\
\text { textiles y acabado de } \\
\text { textiles }\end{array}$ & 8.7 & 8.2 & 8 & 7.7 & 7.1 & 7 & 7.5 & 7.8 & 7.9 \\
\hline $\begin{array}{l}\text { Fabricación de } \\
\text { productos textiles }\end{array}$ & 7.5 & 7.1 & 6.8 & 6.2 & 6 & 5.8 & 7.1 & 6.8 & 7 \\
\hline $\begin{array}{l}\text { Fabricación de prendas } \\
\text { de vestir }\end{array}$ & 6.4 & 6 & 5.8 & 5.8 & 5.6 & 5.4 & 5.6 & 5.8 & 5.9 \\
\hline $\begin{array}{l}\text { Curtido y acabado de } \\
\text { cuero y piel }\end{array}$ & 7.3 & 6.6 & 6.2 & 6.2 & 6.5 & 6.3 & 6.8 & 7.2 & 7.3 \\
\hline Subsector 32 & 15.1 & 14.5 & 14.8 & 14 & 13.1 & 12.5 & 12.8 & 12.9 & 12.7 \\
\hline Industria de la madera & 7.8 & 7.3 & 7.2 & 6.6 & 6.1 & 5.9 & 6.3 & 6.3 & 6.4 \\
\hline Industria del papel & 12.8 & 12.1 & 12 & 11.7 & 11.1 & 10.9 & 11.4 & 11.6 & 11.8 \\
\hline $\begin{array}{l}\text { Impresión e industrias } \\
\text { conexas }\end{array}$ & 9.8 & 9.2 & 9.7 & 9.3 & 8.8 & 8.2 & 8.7 & 9.8 & 10.3 \\
\hline $\begin{array}{l}\text { Fabricación de } \\
\text { productos derivados del } \\
\text { petróleo }\end{array}$ & 36 & 36.4 & 36.3 & 35.6 & 34.8 & 33.5 & 36.5 & 37 & 38 \\
\hline Industria química & 21.8 & 20.6 & 20.9 & 19.7 & 19 & 18.1 & 17.7 & 17.9 & 17.4 \\
\hline $\begin{array}{l}\text { Industria del plástico y } \\
\text { del hule }\end{array}$ & 9.4 & 9 & 8.9 & 8.3 & 7.7 & 7.5 & 8.3 & 8.5 & 8.5 \\
\hline $\begin{array}{l}\text { Fabricación de } \\
\text { productos con minerales } \\
\text { no metálicos }\end{array}$ & 13.2 & 12.7 & 13.1 & 12.6 & 10.8 & 9.9 & 9.3 & 9 & 8.8 \\
\hline Subsector 33 & 11.8 & 11.3 & 11.4 & 10.9 & 10.3 & 9.9 & 10.1 & 10.4 & 10.7 \\
\hline $\begin{array}{l}\text { Industrias metálicas } \\
\text { básicas }\end{array}$ & 15.2 & 14.7 & 14.8 & 14.4 & 14 & 13 & 13.5 & 13.8 & 13.9 \\
\hline $\begin{array}{l}\text { Fabricación de } \\
\text { productos metálicos }\end{array}$ & 9.7 & 9.4 & 9.1 & 9 & 8.2 & 8 & 8.2 & 8.2 & 8.2 \\
\hline $\begin{array}{l}\text { Fabricación de } \\
\text { maquinaria y equipo }\end{array}$ & 13.9 & 13.8 & 13.7 & 13.4 & 12.6 & 12.5 & 12.4 & 12.6 & 12.9 \\
\hline $\begin{array}{l}\text { Fabricación de equipo } \\
\text { de computación, }\end{array}$ & 11.1 & 10.7 & 11.3 & 10.8 & 10.3 & 10 & 10.4 & 11.5 & 11.7 \\
\hline $\begin{array}{l}\text { Fabricación de } \\
\text { accesorios, aparatos } \\
\text { eléctricos }\end{array}$ & 11.4 & 10.9 & 11.4 & 10.6 & 10.1 & 9.7 & 9.9 & 10.2 & 9.9 \\
\hline $\begin{array}{l}\text { Fabricación de equipo } \\
\text { de transporte }\end{array}$ & 12.8 & 12.3 & 12.1 & 11.4 & 10.5 & 9.9 & 10 & 10.2 & 10.7 \\
\hline $\begin{array}{l}\text { Fabricación de muebles, } \\
\text { colchones y persianas }\end{array}$ & 9.4 & 8.7 & 8.7 & 8.5 & 8.3 & 8.1 & 8.3 & 8.6 & 8.7 \\
\hline $\begin{array}{l}\text { Otras industrias } \\
\text { manufactureras }\end{array}$ & 11 & 10.4 & 10.9 & 10.5 & 10.1 & 10 & 10.4 & 10.6 & 11 \\
\hline
\end{tabular}

Fuente: Elaboración propia con datos de la Encuesta Mensual de la Industria Manufacturera (EMIM) del INEGI. 


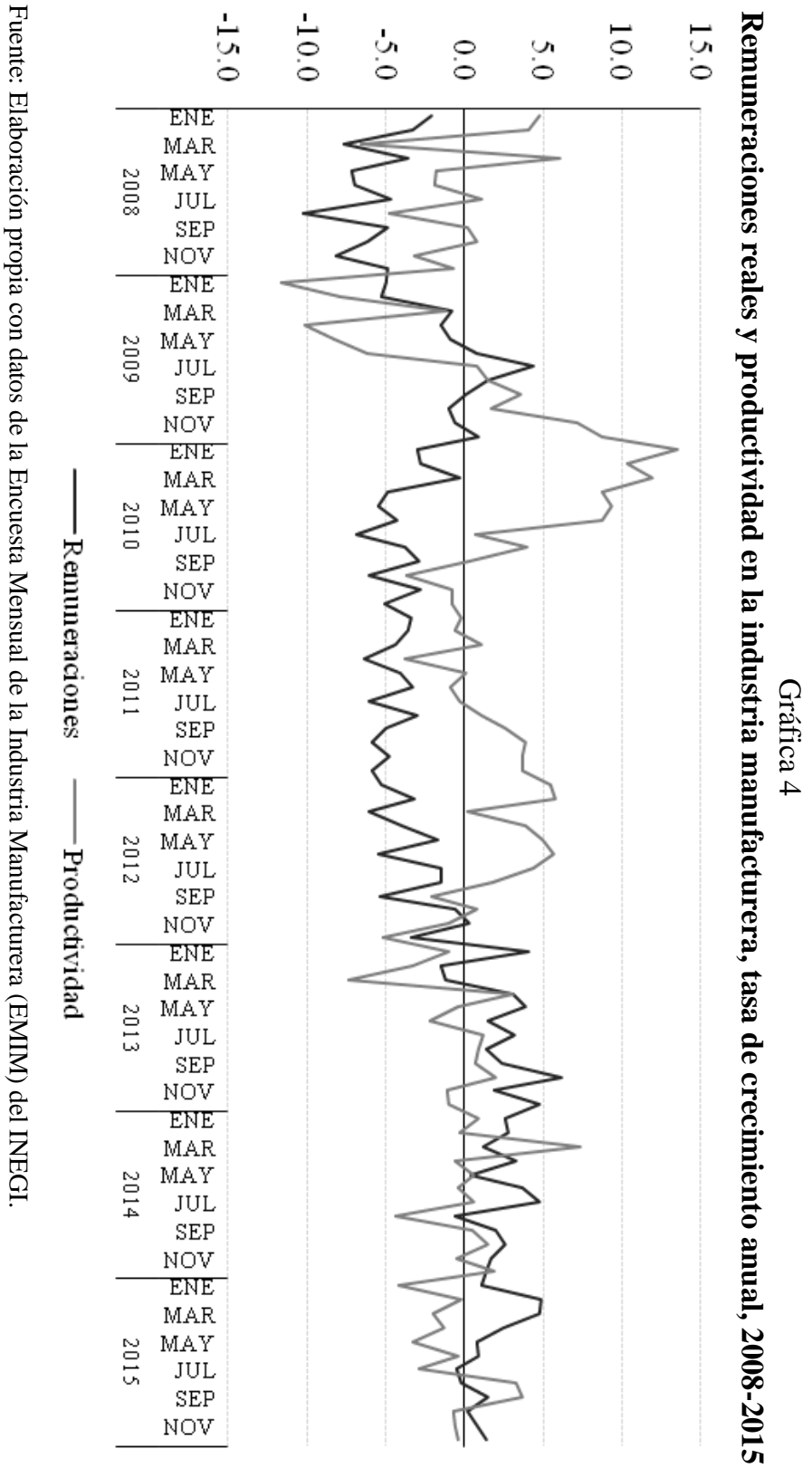


Como lo menciona Mendoza y Martínez (1999), hasta antes de la firma del TLC, el sector manufacturero se vio guiado por un incremento del empleo en la región fronteriza del país comprendida por los estados de Baja California, Sonora, Coahuila, Chihuahua, Nuevo León, Sonora y Tamaulipas. En conjunto, mostraron un crecimiento promedio anual de $5.8 \%$ en el periodo de 1980 a 1994, siendo Baja California, Tamaulipas y Coahuila las más beneficiadas, mientras que entidades no fronterizas crecieron a únicamente $2.5 \%$, cada año. Asimismo, pese a que en los estados no fronterizos las industrias de mayor expansión fueron las relacionadas con el mercado interno, la gran mayoría mantenía un vínculo con actividades orientadas hacia la exportación.

Uno de los elementos que ayuda a explicar la relocalización de las manufacturas al norte del país, se relaciona con el bajo nivel de remuneraciones de la región, pero es precisamente este elemento el que posteriormente sufriría cambios importantes. En particular, la concentración de la manufactura en estados de la frontera norte del país permitió romper, o al menos reducir, las tendencias históricas que dictaban diferencias importantes entre los salarios de la región norte y los salarios de la región centro del país. Las ramas industriales, que en la década de los noventa mantuvieron un vínculo importante con el exterior fueron aquellas que lograron reducir en mayor medida dicha brecha salarial; Chihuahua y Coahuila fueron las de mayor crecimiento; Baja California continuó sin cambios importantes. Entre 2005 y 2014, los estados del norte del país siguieron mostrando crecimiento con una TMCA del PIB del sector de 3.3\%, mientras que para los estados del centro y centro-norte fue de $1.3 \%$ y $2.6 \%$, respectivamente. De igual forma, este patrón se ve reflejado en lo que a empleo se refiere. La región norte fue la única que incrementó su participación con respecto al personal ocupado en el sector, pasando de $24.0 \%$ a $27.7 \%$ de 2005 a 2014; mientras que en el centro se redujo de $41.7 \%$ a $39.8 \%$ (cuadro 5).

Dentro de la región norte, Coahuila es el estado con mayor dinamismo tanto en términos de crecimiento de su producción (4.4\% promedio anual) como de empleo ( $4.5 \%$ promedio anual). Por su parte, Nuevo León y Sonora han crecido a un nivel similar aunque menor al de Coahuila (4.0\% y $4.2 \%$, respectivamente), y dicho crecimiento de la producción se ha reflejado en un menor nivel de crecimiento dentro del empleo manufacturero $(1.2 \%$ y $1.1 \%$, respectivamente). Finalmente, cabe resaltar el caso de la Ciudad de México, siendo esta la entidad con mayor pérdida de empleo en este periodo ( $-4.9 \%$ promedio anual) (cuadro 5). 
Cuadro 5

PIB y personal ocupado sector manufacturero, 2005-2014 (\%)

\begin{tabular}{|c|c|c|c|c|c|c|}
\hline \multirow{3}{*}{ Periodo } & \multicolumn{3}{|c|}{ PIB sector manufacturero } & \multicolumn{3}{|c|}{ Personas ocupadas } \\
\hline & \multicolumn{2}{|c|}{ Distribución } & \multirow{2}{*}{$\begin{array}{l}T M C A \\
2005-2014\end{array}$} & \multicolumn{2}{|c|}{ Distribución } & \multirow{2}{*}{$\begin{array}{l}T M C A \\
2005-2014\end{array}$} \\
\hline & 2005 & 2014 & & 2005 & 2014 & \\
\hline Centro & 41.6 & 38.8 & 1.3 & 41.7 & 39.8 & -0.1 \\
\hline Cdmx & 10.0 & 6.9 & -1.9 & 6.9 & 5.3 & -4.4 \\
\hline Guanajuato & 6.1 & 7.3 & 3.5 & 6.5 & 7.2 & 3.9 \\
\hline Hidalgo & 3.3 & 2.7 & 0.2 & 2.3 & 2.2 & 1.0 \\
\hline México & 12.7 & 11.9 & 1.6 & 15.2 & 15.9 & -0.4 \\
\hline Morelos & 1.3 & 1.5 & 3.9 & 1.2 & 1.1 & 1.2 \\
\hline Puebla & 4.4 & 4.1 & 2.5 & 6.0 & 4.7 & -1.2 \\
\hline Querétaro & 3.0 & 3.6 & 4.2 & 2.1 & 1.9 & 3.4 \\
\hline Tlaxcala & 0.9 & 0.9 & 0.7 & 1.5 & 1.6 & 1.0 \\
\hline Centro-Norte & 16.2 & 15.9 & 2.6 & 20.2 & 19.5 & 1.5 \\
\hline Aguascalientes & 1.4 & 2.4 & 8.8 & 1.2 & 1.4 & 3.4 \\
\hline Baja California Sur & 0.1 & 0.1 & 1.0 & 0.2 & 0.3 & -3.4 \\
\hline Colima & 0.2 & 0.2 & 0.5 & 0.4 & 0.4 & 3.7 \\
\hline Durango & 1.5 & 1.3 & 0.5 & 1.4 & 1.4 & 1.4 \\
\hline Jalisco & 7.2 & 7.3 & 2.4 & 8.3 & 7.5 & 0.1 \\
\hline Michoacán & 2.6 & 2.1 & 0.3 & 3.6 & 3.1 & 0.6 \\
\hline Nayarit & 0.2 & 0.2 & 1.9 & 0.5 & 0.5 & 1.6 \\
\hline San Luis Potosí & 2.7 & 3.0 & 3.0 & 2.3 & 2.5 & 3.4 \\
\hline Sinaloa & 1.2 & 1.1 & 1.5 & 1.7 & 1.7 & 0.6 \\
\hline Zacatecas & 0.5 & 0.5 & 3.9 & 0.6 & 0.8 & 9.7 \\
\hline Norte & 28.9 & 32.0 & 3.3 & 24.0 & 27.7 & 1.7 \\
\hline Baja California & 3.6 & 3.3 & 1.5 & 3.8 & 4.8 & -3.4 \\
\hline Coahuila & 6.7 & 8.2 & 4.4 & 3.4 & 4.4 & 4.5 \\
\hline Chihuahua & 3.5 & 3.5 & 2.7 & 4.3 & 5.2 & 1.9 \\
\hline Nuevo León & 9.0 & 10.5 & 4.0 & 6.2 & 6.5 & 1.2 \\
\hline Sonora & 2.9 & 3.3 & 4.2 & 2.4 & 2.8 & 1.1 \\
\hline Tamaulipas & 3.1 & 3.1 & 1.9 & 3.8 & 3.9 & 1.3 \\
\hline Sur & 11.9 & 10.9 & 1.2 & 14.1 & 13.0 & -1.0 \\
\hline Campeche & 0.1 & 0.1 & 1.4 & 0.5 & 0.5 & -2.7 \\
\hline Chiapas & 0.9 & 0.8 & 0.2 & 1.7 & 1.6 & 1.0 \\
\hline Guerrero & 0.5 & 0.4 & 1.0 & 1.6 & 1.7 & -1.5 \\
\hline Oaxaca & 1.6 & 1.3 & 0.2 & 3.0 & 2.3 & -2.4 \\
\hline Quintana Roo & 0.3 & 0.3 & 2.2 & 0.5 & 0.4 & -3.5 \\
\hline Tabasco & 1.1 & 0.9 & -0.6 & 0.6 & 0.7 & -1.3 \\
\hline Veracruz & 6.1 & 5.9 & 1.9 & 3.9 & 3.7 & 0.0 \\
\hline Yucatán & 1.4 & 1.3 & 1.5 & 2.3 & 2.1 & -2.0 \\
\hline
\end{tabular}

Fuente: Elaboración propia con datos de la Encuesta Mensual de la Industria Manufacturera (EMIM), la Encuesta Nacional de Ocupación y Empleo (ENOE) y el Sistema de Cuentas Nacionales de México del INEGI. * Para la regionalización se consideró la clasificación que realiza el Banco de México en el Reporte Sobre las Economías Regionales. 
El comportamiento antes descrito puede ser explicado por el crecimiento que han tenido las ramas correspondientes a la fabricación de maquinaria y equipo, equipo de cómputo, componentes y accesorios electrónicos y aparatos eléctricos, ramas estrechamente relacionadas con el comercio exterior. En promedio, dichas actividades representan cerca del $41.6 \%$ del total de la actividad manufacturera de cada entidad fronteriza, y sus tasas de crecimiento promedio fueron de 1.6, 2.9 y 3.7 por ciento para Baja California, Chihuahua y Tamaulipas, respectivamente, y de 8.0, 8.4 y 11.6 por ciento en Coahuila, Nuevo León y Sonora.

En relación con el mercado laboral del sector manufacturero, pese a que las brechas salariales del dicho sector entre la región norte y la región del centro se han reducido desde la década de los ochenta, y aunque en la mayor parte de las industrias de maquinaria y equipo, equipo eléctrico y equipo electrónico han experimentado un crecimiento importante, en general, las remuneraciones en el centro del país siguen siendo superiores a las de los estados colindantes con el país vecino del norte. Como se observa en la gráfica 5, de 2007 a 2015, en promedio en el centro del país, las remuneraciones mensuales ascendieron a 12 mil 382 pesos; mientras que en la región norte se situaron ligeramente por debajo, en 11 mil 932 pesos. Cabe señalar que dentro de las entidades que comprenden la región sur (Campeche), en promedio las remuneraciones tienen un nivel superior al resto de las regiones, sin embargo esto se explica por el efecto de la industria petrolera.

\section{Estrategia metodológica}

\subsection{Metodología de análisis}

El mercado laboral mexicano está caracterizado por la rigidez de los salarios, por lo que los supuestos neoclásicos que describen un mercado laboral en competencia perfecta resultan insuficientes. Particularmente, la rigidez en los salarios en México se relaciona con la distorsión del nivel de desempleo que se ve afectado por la informalidad laboral, el flujo migratorio de trabajadores mexicanos hacia los Estados Unidos y el efecto derivado de la apertura comercial para mantener el bajo costo de mano de obra para promover la Inversión Extranjera Directa (IED). Así, el crecimiento de los salarios se encuentra limitado a la dependencia que tenga la economía con respecto a la inversión proveniente del exterior y el ambiente institucional de fijación del salario mínimo. 
Gráfica 5

Remuneraciones mensuales sector manufacturero, promedio 20072015 (Pesos)

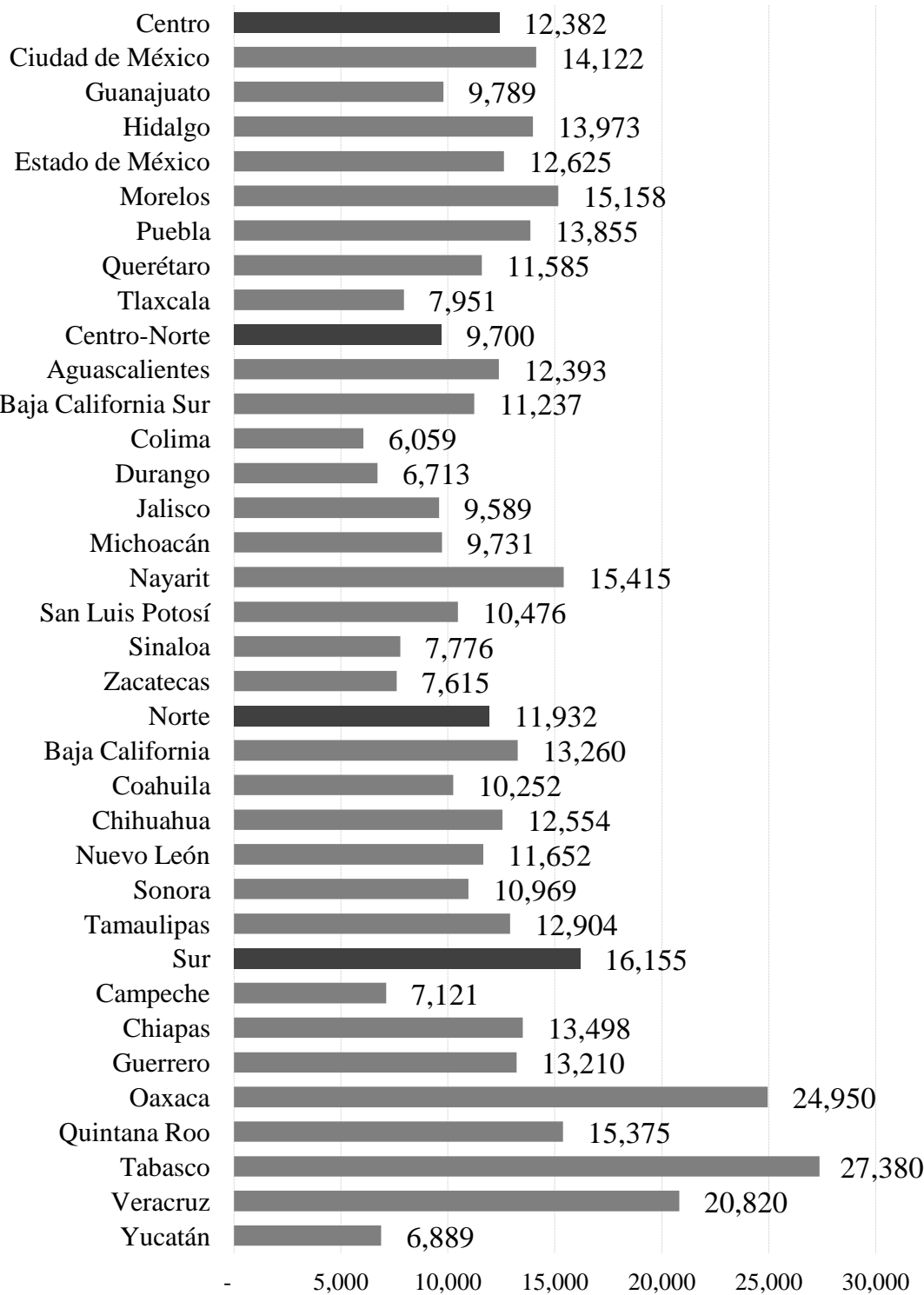

Fuente: Elaboración propia con datos de la Encuesta Mensual de la Industria Manufacturera (EMIM) y el Sistema de Cuentas Nacionales de México del INEGI. 
En la presente investigación, se propone un análisis para el caso del sector manufacturero mexicano. El enfoque teórico y empírico en que se fundamenta el análisis es aquel que incluye a la productividad de los trabajadores como parte de la actual política laboral para el incremento de los salarios $^{2}$. Asimismo, se incorpora el comportamiento del desempleo como principal indicador del mercado laboral; el nivel de precios como referente del poder adquisitivo de las remuneraciones reales; y el nivel de salario mínimo, que pese a establecerse de manera institucional, es el principal indicador dentro de las negociaciones salariales.

Para un análisis en el nivel agregado, se propone la siguiente ecuación de salarios:

$$
w_{i t}=\alpha_{i}+\beta_{1} \varphi_{i, t}+\beta_{2} U n_{i, t}+\beta_{3} S M_{i, t}+\beta_{4} \pi_{i, t}+\varepsilon_{i, t}
$$

donde $w_{i, t}$ es el logaritmo del salario real en la entidad $i$, para el periodo $t$; $\varphi_{i, t}$, el logaritmo de la productividad laboral en la entidad $i$, para el periodo $t$ $U n_{i, t}$, el logaritmo de la tasa de desempleo en la entidad $i$, para el periodo $t$; $S M_{i, t}$, el logaritmo de la inflación en la entidad $i$, para el periodo $t$, y $\varepsilon_{i, t}$ representa el término error.

En la ecuación 1, se incorporan efectos espaciales y temporales, considerando que, en diferentes entidades para el caso de México, es posible observar características particulares que no son posibles de capturar con un solo intercepto, como usualmente se realiza en el análisis de series de tiempo. La especificación de la ecuación corresponde a elasticidades entre los salarios y cada una de las variables sugeridas en los trabajos empíricos antes mencionados.

Para la estimación de la ecuación, el análisis se limita al periodo 2007-2015, considerando la información disponible en encuestas especializadas para el tema, como la Encuesta Mensual de la Industria Manufacturera (EMIM) y la Encuesta Nacional de Ocupación y Empleo (ENOE). En el primer caso, particularmente, pese a tener como antecedente a la Encuesta Industrial Mensual (EIM) que data desde 1964, se han realizado cambios metodológicos significativos en diversos periodos, de manera que este lapso es el más reciente y consistente. Es digno de resaltar también, que en este se integra la operación de la Industria Maquiladora de Exportación y la de Importación Temporal para Producir Artículos de Exportación, con el fin de realizar una medición más integral del sector manufacturero (INEGI, 2016).

\footnotetext{
${ }^{2}$ En lo consecuente, de no especificarse lo contrario, el término salarios refiere a salarios reales. Igualmente, la productividad laboral refiere a términos reales.
} 


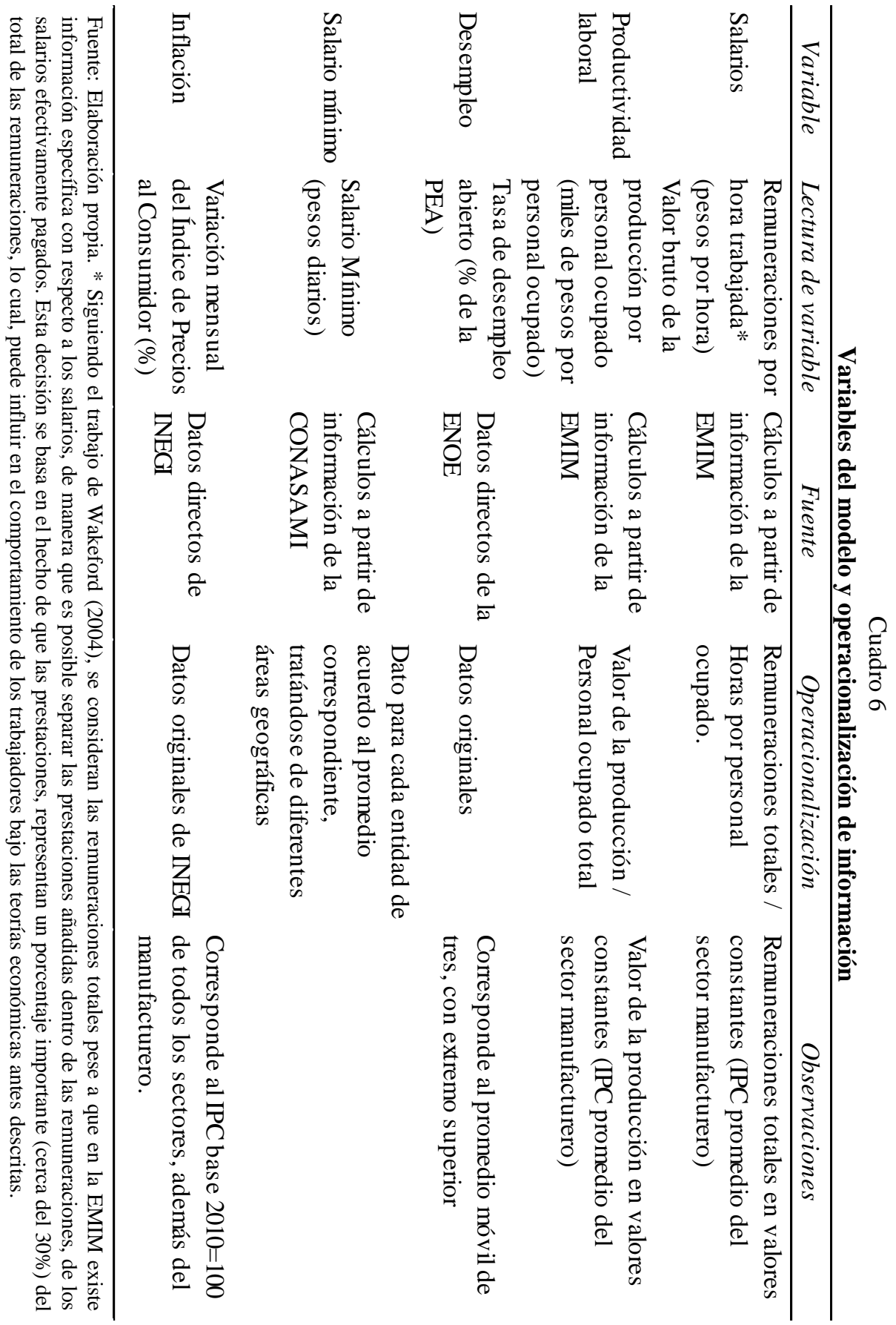


A continuación, en el cuadro 6, se muestran cada una de las variables utilizadas, el nombre utilizado, la lectura que se debe dar a estas, así como su correspondiente descripción; además de la forma en que fueron calculadas, las fuentes oficiales de donde procedieron y de ser requerido, algunas observaciones que clarifiquen su uso

Se destaca que el salario promedio por hora, en las entidades, sea de cerca de $\$ 51.7$ pesos por hora (cuadro 7), que se traducen en $\$ 12$ mil 408 pesos mensuales. Como se observa, existe una dispersión importante entre el valor mínimo y el valor máximo, que como se mencionó anteriormente, se debe a los altos salarios en algunos estados que mantienen actividades petroleras. Para el caso de la productividad laboral, en promedio para cada entidad, el valor de la producción añadido por cada trabajador fue de $\$ 184.5$ mil pesos en cada mes, igualmente la dispersión entre el valor máximo y el valor mínimo es considerable y se explica de la misma forma que para el caso de los salarios.

\section{Cuadro 7}

Características generales de los datos, 2007-2015

\begin{tabular}{lccccc}
\hline Indicador & Salarios & $\begin{array}{l}\text { Productivi } \\
\text { dad }\end{array}$ & Desempleo & $\begin{array}{c}\text { Salario } \\
\text { Mínimo }\end{array}$ & Inflación \\
\hline $\begin{array}{l}\text { Promedio } \\
\text { Valor }\end{array}$ & 51.7 & 184.5 & 4.5 & 57.3 & 0.3 \\
$\begin{array}{l}\text { Máximo } \\
\text { Valor }\end{array}$ & 221.0 & $1,341.6$ & 9.6 & 70.1 & 9.9 \\
$\begin{array}{l}\text { Mínimo } \\
\text { Desviación }\end{array}$ & 18.8 & 10.2 & 0.7 & 47.6 & -8.5 \\
$\begin{array}{l}\text { Estándar } \\
\text { Obs. }\end{array}$ & 21.9 & 183.0 & 1.6 & 6.4 & 0.9 \\
\hline
\end{tabular}

Fuente: Elaboración propia.

En la gráfica 6a, se resalta la tendencia de los datos considerados como un conjunto sin distinción entre entidades, y se muestra una relación positiva entre salarios y productividad laboral. Sin embargo, al considerar la tendencia particular para cada entidad (gráfica 6b), únicamente seis estados muestran un comportamiento positivo entre salarios y desempleo: Baja California Sur, Colima, Michoacán, Campeche, Jalisco y Oaxaca.

De las entidades restantes, Hidalgo, Querétaro, Veracruz, Aguascalientes, Morelia y Nayarit no parecen tener una relación clara, siendo que los salarios prácticamente no muestran cambios ante diferentes niveles de productividad. Finalmente, las 20 entidades restantes mantienen una tendencia negativa claramente definida. 


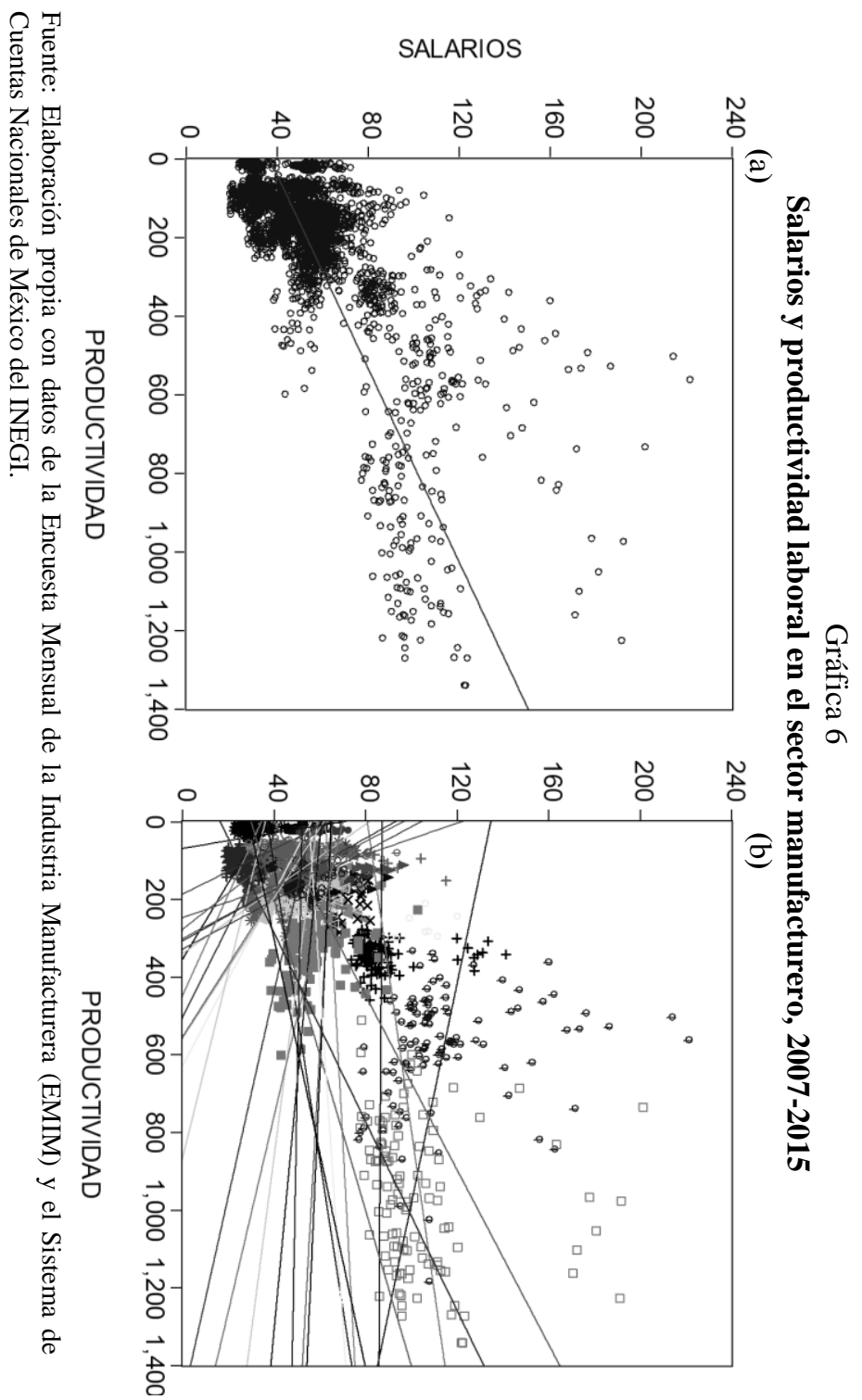


La estrategia econométrica se basa en la estimación de estructuras estáticas, dinámicas y cointegración de largo plazo, como a continuación se describe. El análisis estático partirá del análisis y elección del modelo de efectos fijos y el modelo de efectos aleatorios. En el primer caso, se considera que los regresores impactan de igual manera en cada unidad de corte transversal y que las características individuales entre ellas son definidas por el intercepto. En el modelo de efectos aleatorios, se considera el impacto de los regresores pero también las características individuales, para cada unidad, de corte transversal. Es decir, se considera a $\alpha_{i}$ como un error específico de grupo similar a $\varepsilon_{i t}$, excepto que, para cada unidad de corte transversal, permanece constante a lo largo del tiempo.

Tomando en consideración que la estructura del panel de información refiere a un panel largo vinculado con datos poblacionales, el modelo de efectos fijos parece representar la mejor opción. No obstante lo anterior, pruebas estadísticas como la prueba Breush-Pagan y la prueba Hausman permiten obtener criterios más precisos y concluyentes.

En el modelo estático, se asume la ausencia de ajustes en los salarios a través del tiempo, lo cual define un modelo no dinámico que supone un papel nulo de las negociaciones salariales entre trabajadores y empleadores.

Para el análisis dinámico, la ecuación 2 replantea el análisis de los salarios, bajo el supuesto en el cual los salarios del periodo $t$ son afectados adicionalmente por los salarios negociados en un periodo anterior $k$, no mayor a un año. Esto último, considerando que las negociaciones salariales suelen ocurrir una vez cada año.

$$
w_{i, t}=\alpha_{i}+\beta_{0} w_{i, t-k}+\beta_{1} \varphi_{i, t}+\beta_{2} U n_{i, t}+\beta_{3} S M_{i, t}+\beta_{4} \pi_{i, t}+\varepsilon_{i, t}
$$

donde $0<k<12$, tratándose de datos mensuales, como es el caso. La dinámica de la ecuación se presenta una vez incorporada la variable dependiente en forma retardada $w_{i, t-k}$, quedando el resto de las variables en su forma original, correspondiente a la ecuación 1.

En términos metodológicos, esta incorporación complica el modelo debido a la presencia de dos fuentes de persistencia a lo largo del tiempo: la autocorrelación entre la variable retardada y los regresores, así como la autocorrelación asociada con los efectos individuales y el término de error (Baltagi, 2005). Con lo anterior, la aplicación de métodos como MCO o efectos fijos para un panel dinámico, genera estimaciones sesgadas e inconsistentes. Al respecto, Arellano y Bond (1991) proponen el Método Generalizado de Momentos (MGM), con el cual resulta eficiente el uso de los rezagos de la variable dependiente. 
Por otra parte, cabe señalar que la longitud del panel impone restricciones siendo que el método de MGM es sugerido principalmente para paneles cortos. De forma práctica, Álvarez y Arellano (2003) establecen que los estimadores obtenidos a partir de MGM, son consistentes cuando $T / N \rightarrow C$ para $0<c<2$. Para los datos en esta investigación se tiene $T=108$ meses y $N=32$ dando un valor $c=3.375$ con lo cual se violaría esta condición.

Para el cumplimiento de dicha condición, de manera alternativa, cada serie mensual de cada entidad puede ser transformada en serie trimestral, si se toman los meses centrales de cada trimestre como referencia. Esta operación no modifica el comportamiento de las series, y se cumple con la condición de Álvarez y Arellano (2003), siendo que ahora $T=36$ meses y $N=32$, dando un valor $c=1.125$.

Para validar la consistencia de los estimadores, se propone evaluar los supuestos del modelo: los residuos están intercorrelacionados y las variables explicativas son exógenas. Los contrastes de sobreidentificación de Sargan y las pruebas de correlación serial residual de primero y segundo orden de Arellano y Bond, permiten corroborar dichas condiciones.

Finalmente, para evaluar la relación de largo plazo entre las variables y complementar el análisis de la ecuación 2, se propone un análisis de cointegración de panel. Esto a su vez permite asegurar que las relaciones encontradas en el modelo estático y dinámico no resulten espurias, al asumir el factor estacionario en variables económicas. Para este último tipo de análisis, en primer lugar, se evalúan las condiciones de estacionariedad de las variables a través de pruebas de raíz unitaria, con la ventaja de que las pruebas basadas en paneles tienen mayor potencia que las pruebas basadas en series de tiempo; en segundo lugar, se determina si existe una relación de equilibrio a largo plazo entre la inflación, la productividad y los salarios reales, utilizando el test de cointegración de panel sugerido por Pedroni (1999); y en tercer lugar, se estiman los coeficientes de largo plazo mediante la metodología para datos panel Fully-Modified Ordinary Least Squares (FMOLS) y Dynamic Ordinary Least Squares (DOLS).

En cuanto a las condiciones de estacionariedad, se evalúa la presencia de raíces unitarias para las tres variables usando pruebas para datos de tipo panel como las establecidas por Breitung (2001), Levin et al. (2002), e Im et al. (2003), basados en el test Augmented Dickey Fuller (ADF); mientras Maddala y Wu (1999), Choi (2001) y Hadri (2000) utilizan un test del tipo Phillip-Perron (PP).

De manera formal, la especificación de las pruebas se observa mediante el análisis de las restricciones que pudieran existir entre las secciones 
transversales, considerando procesos autorregresivos $\mathrm{AR}(1)$, como los siguientes:

$$
\begin{aligned}
& y_{i, t}=\rho_{i} y_{i, t-1}+\delta X_{i, t}+\varepsilon_{i, t} \\
& y_{i, t}=\alpha_{i}+\rho_{i} y_{i, t-1}+\delta X_{i, t}+\varepsilon_{i, t} \\
& y_{i, t}=\alpha_{i}+\beta_{i} t+\rho_{i} y_{i, t-1}+\delta X_{i, t}+\varepsilon_{i, t}
\end{aligned}
$$

donde $y_{i, t}$ es la variable explicada, en este caso, los salarios; $x_{i, t}$, las variables exógenas para control de efectos fijos de grupo y tendencias en el tiempo; $t$ es la variable de tiempo o de tendencia; el término de error está representado por $\varepsilon_{i, t}$; y $\rho_{i}$ son los coeficientes autorregresivos. Bajo esta especificación, si $|\rho|<1, y_{i}$ se considerará como un proceso débilmente estacionario, y si $|\rho|=1, y_{i}$ contará con raíz unitaria.

Cabe señalar que las pruebas de Breitung (2001), Levin et al. (2002), y Hadri (2000) tienen como limitante el hecho de asumir que existe un proceso común de raíz unitaria para todas las secciones transversales; es decir, $\rho_{i}=\rho$ para cada $i$, de lo cual, se infiere que cada una de las 32 entidades del modelo converjan hacia el equilibrio de largo plazo, a una misma velocidad. En respuesta a esta limitante, Im et al. (2003), Maddala y Wu (1999) y Choi (2001) eliminan este supuesto permitiendo un $\rho_{i}$ para cada sección cruzada, estimando la regresión ADF en forma separada. Con esto, Breitung (2001) y Levin et al. (2002), evalúan el proceso bajo la hipótesis nula $\alpha=0$ de raíz unitaria para todos los procesos individuales y la hipótesis alternativa $\alpha<$ 0 de no raíz unitaria para ningún proceso individual.

En forma opuesta, Hadri (2000) utiliza una hipótesis nula de no raíz unitaria para todos los procesos individuales y una alternativa de raíz unitaria para todos los procesos individuales. Por su parte, Im et al. (2003), Maddala y Wu (1999) y Choi (2001) evalúan bajo la hipótesis nula $\alpha_{i}=0$ de raíz unitaria en todas las secciones transversales, pero con una hipótesis alternativa de no raíz unitaria en algunas secciones transversales, siendo $\alpha_{i}=0$ para $i=$ $1,2, \ldots, N$ y $\alpha_{i}<0$ para $i=N+1, N+2, \ldots, N$.

Una vez definidas las condiciones de estacionariedad entre salarios, productividad laboral y desempleo, con base en las pruebas anteriores, se procede a realizar la prueba de cointegración de panel sugerida por Pedroni (1999), misma que representa una extensión de las pruebas de Engle y Granger (1987) con el fin de incluir datos tipo panel. A diferencia de la prueba para series de tiempo, en datos tipo panel se asume que el término autorregresivo de primer orden es el mismo para todas las secciones transversales, mientras que los interceptos y los coeficientes de tendencia pueden variar. 
Como paso final, solo después de corroborar que existe un vector de cointegración y que hay una relación de equilibrio de largo plazo, se procede a obtener los estimadores de largo plazo mediante la técnica Fully Modify Ordinary Least Squares (FMOLS) de Phillips y Hansen (1990), a través de un sistema de ecuaciones para un panel cointegrado como sigue:

$y_{i, t}=\alpha_{i, t}+\beta X_{i, t}^{\prime}+\varepsilon_{i, t}$

donde los regresores $x_{i, t}$ son procesos integrados de orden 1 para todo $i$, siendo

$$
x_{i, t}=x_{i, t-1}+u_{i, t}
$$

De manera complementaria, se propone el estimador bajo la técnica Dynamic Ordinary Least Squares (DOLS) de Saikkonen (1992) y Stock y Watson (1993), el cual trabaja en forma similar al FMOLS, siendo que además de los rezagos utilizados para $X_{i, t}^{\prime}$, también se agregan un número $t+q$ de adelantos $\Delta x_{i, t+j}^{*}$ de los regresores en sus primeras diferencias, para eliminar la endogeneidad asintótica. De esta manera, el sistema queda:

$y_{i, t}=\alpha_{i, t}+\beta X_{i, t}^{\prime}+\sum_{j=-q}^{q} c_{i, j} \Delta x_{i, t+j}^{*}+\varepsilon_{i, t}$

Asimismo, el método DOLS supone que la aplicación de los rezagos y adelantos de los regresores en el sistema absorberá toda la correlación de largo plazo entre los errores de los estimadores. Autores como Kao y Chiang (2001) y Wanger y Hlouskova (2007) sugieren que los estimadores DOLS representan una mejor alternativa que los estimadores FMOLS, tratándose de paneles cointegrados y/o que pudieran presentar problemas de correlación en corte trasversal.

\subsection{Resultados}

El cuadro 8 muestra los resultados de la ecuación de salarios mediante diferentes métodos de estimación, para corroborar la heterogeneidad en el comportamiento de cada entidad, en cuanto se refiere a salarios y sus determinantes. La primera columna corresponde a la estimación de la ecuación 1, a través de Mínimos Cuadrados Ordinarios (MCO). Las dos columnas siguientes corresponden a la estimación mediante el Modelo de Efectos Fijos (MEF1) y el Modelo de Efectos Aleatorios (MEA). Finalmente, para dar solución a posibles problemas de autocorrelación y heterocedasticidad, las últimas dos columnas corresponden a la estimación del Modelo de Efectos Fijos con Errores Estándar Robustos (MEF2) y con el Modelo de Mínimos Cuadrados Ponderados (MCP), respectivamente. 
Cuadro 8

Estimación general de la ecuación de salarios, 2007.01-2015.12

Variable dependiente: salarios (lnw)

\begin{tabular}{|c|c|c|c|c|c|}
\hline $\begin{array}{l}\text { Variable } \\
\text { independiente }\end{array}$ & $\begin{array}{l}\text { (a) } \\
M C O\end{array}$ & $\begin{array}{l}(b) \\
M E F 1\end{array}$ & $\begin{array}{l}(c) \\
M E A\end{array}$ & $\begin{array}{l}\text { (d) } \\
M E F 2\end{array}$ & $\begin{array}{l}\text { (e) } \\
M C P^{I /}\end{array}$ \\
\hline Intercepto & $\begin{array}{c}4.3811 * * * \\
(0.1118)\end{array}$ & $\begin{array}{c}4.2757 * * * \\
(0.1100)\end{array}$ & $\begin{array}{c}4.2229 * * * \\
(0.1221)\end{array}$ & $\begin{array}{c}4.2757 * * * \\
(0.3381)\end{array}$ & $\begin{array}{c}3.2007 * * * \\
(0.1399)\end{array}$ \\
\hline $\begin{array}{l}\text { Productividad } \\
(\ln \varphi)\end{array}$ & $\begin{array}{c}-0.0787 * * * \\
(0.0142)\end{array}$ & $\begin{array}{c}-0.0787 * * * \\
(0.0142)\end{array}$ & $\begin{array}{c}-0.0640 * * * \\
(0.0139)\end{array}$ & $\begin{array}{c}-0.0787 * * \\
(0.0389)\end{array}$ & $\begin{array}{c}0.2177 * * * \\
(0.0060)\end{array}$ \\
\hline $\begin{array}{l}\text { Desempleo } \\
(\ln U n)\end{array}$ & $\begin{array}{c}-0.0371 * * * \\
(0.0120)\end{array}$ & $\begin{array}{c}-0.0371 * * * \\
(0.0120)\end{array}$ & $\begin{array}{c}-0.0370 * * * \\
(0.0120)\end{array}$ & $\begin{array}{l}-0.0371 * \\
(0.0222)\end{array}$ & $\begin{array}{c}-0.0236 * * \\
(0.0098)\end{array}$ \\
\hline Inflación $(\ln \pi)$ & $\begin{array}{c}0.0093 * * * \\
(0.0026)\end{array}$ & $\begin{array}{c}0.0093 * * * \\
(0.0026)\end{array}$ & $\begin{array}{c}0.0093 * * * \\
(0.0026)\end{array}$ & $\begin{array}{c}0.0093 * * * \\
(0.0031)\end{array}$ & $\begin{array}{c}0.0061 \\
(0.0038)\end{array}$ \\
\hline $\begin{array}{l}\text { Salario Mínimo } \\
(\ln S M)\end{array}$ & $\begin{array}{r}0.0106 \\
(0.0253) \\
\end{array}$ & $\begin{array}{r}0.0106 \\
(0.0253) \\
\end{array}$ & $\begin{array}{r}0.0068 \\
(0.0254) \\
\end{array}$ & $\begin{array}{r}0.0106 \\
(0.0817) \\
\end{array}$ & $\begin{array}{c}-0.0868 * * \\
(0.0348) \\
\end{array}$ \\
\hline Periodos & 108 & 108 & 108 & 108 & 108 \\
\hline Entidades & 32 & 32 & 32 & 32 & 32 \\
\hline Observaciones ${ }^{\mathrm{a} /}$ & 2900 & 2900 & 2900 & 2900 & 2900 \\
\hline R-cuadrado & 0.870 & 0.870 & n.a. & 0.870 & 0.312 \\
\hline
\end{tabular}

Fuente: Elaboración propia. Notas: Los errores estándar de los coeficientes se muestran entre paréntesis. 1/ Ponderaciones basadas en varianzas de los errores por unidad. *Coeficiente significativo al $10 \%$; **coeficiente significativo al $5 \%$; ***coeficiente significativo al $1 \%$. a/ La presencia de valores negativos en las observaciones para la variable inflación impide la aplicación de logaritmos, lo cual reduce el número de observaciones de 3,456 a 2,903. Seguido a lo anterior, para la variable desempleo, INEGI omite las cifras de Tabasco, correspondientes a noviembre y diciembre de 2007 y enero de 2008.

Con los resultados anteriores, es posible destacar los siguientes aspectos:

- Tanto la productividad como el desempleo resultan significativos para explicar a los salarios. En cuanto al desempleo, en todos los modelos utilizados se comprueba la relación negativa con una elasticidad promedio de 0.03. Para el caso de la productividad, sin considerar el modelo MCP, se observa que el impacto hacia los salarios es negativo, aunque resulta reducido, igual que el desempleo, aproximándose a 0.07 .

- La inflación impacta positivamente a los salarios con una elasticidad de 0.009 , considerando un promedio mensual de inflación del 3\%, lo cual permite explicar la constante pérdida del poder adquisitivo de los salarios desde hace varias décadas.

- Los resultados sugieren que el comportamiento del salario mínimo no incide en los salarios del sector manufacturero. 
Considerando el signo y el nivel de elasticidad en la relación salariosdesempleo, los resultados convergen con los obtenidos por Castro (2006), aun tomando en cuenta que este último se basó en un enfoque microeconómico, mientras que la presente investigación refiere a un nivel mayormente agregado. Esto deriva en que, por medio del análisis estático, se corrobora la hipótesis de la curva de salarios para el caso de México.

Las pruebas de Hausman y Breush-Pagan confirman que el modelo que mejor se ajusta a los datos es el de efectos fijos. Con las estimaciones de efectos fijos y errores estándar robustos (MEF2), la significancia de los coeficientes no es alterada. Los resultados muestran un cambio de signo de la variable productividad. Considerando que estos elementos debilitan una validez completa de los resultados para el análisis estático, resulta importante la evaluación desde una perspectiva dinámica o de largo plazo, que se muestra más adelante.

De forma adicional, dentro del análisis estático en el cuadro 9, se definen cuatro regresiones alternativas para diferentes muestras considerando controles tanto temporales como espaciales. Las primeras dos columnas, respectivamente, corresponden a una muestra para el periodo que involucra la crisis económica (2007.01-2009.06) y una muestra para el periodo posterior de mayor estabilidad (2009.07-2015.12); mientras que las dos columnas restantes corresponden a una agrupación de las entidades en donde, según el análisis exploratorio, existe un grupo de 22 estados con una relación salariosproductividad negativa (columna h) y 10 estados restantes con una relación positiva (columna $i$ ).

Primeramente, se muestra que la inflación mantiene su significancia de forma independiente al periodo comprendido o la región definida, en cualquier caso mantiene una relación positiva y reducida como fue descrito anteriormente. Igualmente, con respecto a la productividad, esta mantiene su significancia para cualquier caso pero en mayor medida para la muestra que integra a las 22 entidades que mantuvieron una relación negativa entre salarios y productividad, cuyo coeficiente incluso se incrementa a 0.18. Caso contrario, los estados con una relación positiva tienen un impacto menor y se reduce su significancia, lo cual va acorde con lo esperado, considerando que este grupo lo integran estados cuya actividad manufacturera no tiene una relación importante dentro de la actividad económica general. Por su parte, en el caso del desempleo, resulta significativo únicamente cuando la economía se encuentra en un periodo relativamente estable. Resulta interesante observar que el desempleo no es significativo al tomar en cuenta solo a entidades con intercambio negativo entre salarios y productividad. 
Cuadro 9

Estimación de la ecuación de salarios con controles temporales y espaciales. Variable dependiente: salarios $(\ln w)$

\begin{tabular}{lllll}
\hline Variable independiente & $(f)$ & $(g)$ & $(h)$ & $(i)$ \\
\hline Intercepto & $3.7193^{* * *}$ & $4.7690^{* * *}$ & $4.6902^{* * *}$ & $3.6681^{* * *}$ \\
& $(0.9438)$ & $(0.4668)$ & $(0.4320)$ & $(0.6454)$ \\
Productividad $(\ln \varphi)$ & $-0.0687^{*}$ & $-0.0731^{* *}$ & $-0.1811^{* * *}$ & $0.0854^{*}$ \\
& $(0.0357)$ & $(0.0356)$ & $(0.0595)$ & $(0.0468)$ \\
Desempleo $(\ln U n)$ & 0.0149 & $-0.1307 * * *$ & -0.0153 & $-0.0917 * *$ \\
& $(0.0208)$ & $(0.0363)$ & $(0.0211)$ & $(0.0376)$ \\
Inflación $(\ln \pi)$ & $0.0087^{*}$ & $0.0077 * *$ & $0.0099 * * *$ & $0.0119 * * *$ \\
& $(0.0050)$ & $(0.0030)$ & $(0.0036)$ & $(0.0038)$ \\
Salario Mínimo $(S M)$ & 0.1261 & -0.0826 & 0.0182 & -0.0218 \\
& $(0.2333)$ & $(0.0906)$ & $(0.0710)$ & $(0.1801)$ \\
\hline Periodos & 30 & 78 & 108 & 108 \\
Entidades & 32 & 32 & 22 & 10 \\
Observaciones & 832 & 2068 & 2012 & 898 \\
R-cuadrado & 0.8825 & 0.8879 & 0.8782 & 0.8656 \\
\hline
\end{tabular}

Fuente: Elaboración propia. Notas: Los errores estándar de los coeficientes se muestran entre paréntesis. Todas las variables son expresadas en logaritmos. *Coeficiente significativo al 10\%; **coeficiente significativo al 5\%; ***coeficiente significativo al $1 \%$.

Dichas diferencias en las elasticidades salario-desempleo limitan la comprobación de la curva de salarios como una ley empírica, que no depende de factores institucionales, del tiempo o de la región. Para el caso de México existen diferencias importantes dependiendo de la estabilidad de la economía y del tipo de sector económico. En este caso, la productividad de los trabajadores es más significativa que la tasa de desempleo para determinar los salarios en entidades cuya economía se caracteriza por el estrecho vínculo con el sector manufacturero. De hecho, al mantenerse negativo dicho coeficiente, nuevamente podría definir argumentos a favor de la hipótesis que indica que el costo de mano de obra diverge de la productividad por representar el principal elemento para atraer IED.

En el cuadro 10, se presentan los resultados de la estimación del análisis dinámico, tomando la especificación de la ecuación 2 , y considerando al conjunto de las 32 entidades federativas, para 36 periodos trimestrales que van de 2007.01 a 2015.04. Los resultados son presentados para diferentes métodos de estimación, donde la columna a y b representan las estimaciones a través de MGM de una etapa que utiliza el estimador en diferencias, para el primer caso (MGM1-DIFF); y el estimador en sistemas, para el segundo (MGM1-SYS). Las columnas c y d, por su parte, corresponden igualmente al 
estimador en diferencias para el primer caso, y al estimador en sistemas para el segundo, pero ahora a partir de MGM en dos etapas.

Cuadro 10

Estimación de la ecuación de salarios con rezagos.

Variable dependiente: salarios ( $\Delta w)$

\begin{tabular}{|c|c|c|c|c|}
\hline \multirow{2}{*}{$\begin{array}{l}\text { Variable } \\
\text { independiente }\end{array}$} & (a) & (b) & (c) & (d) \\
\hline & $\begin{array}{l}M G M 1- \\
D I F F\end{array}$ & $M G M 1-S Y S$ & $\begin{array}{l}M G M 2- \\
D I F F\end{array}$ & $M G M 2-S Y S$ \\
\hline \multirow[t]{2}{*}{ Salarios $(\Delta w(-1))$} & -0.0418 & $0.1018 * * *$ & -0.0378 & $0.0931 * * *$ \\
\hline & $(0.0623)$ & $(0.0335)$ & $(0.0674)$ & $(0.0333)$ \\
\hline \multirow[t]{2}{*}{ Salarios $(\Delta w(-2))$} & 0.0165 & $0.0587^{*}$ & 0.0066 & 0.0493 \\
\hline & $(0.0628)$ & $(0.0312)$ & $(0.0750)$ & $(0.0384)$ \\
\hline \multirow[t]{2}{*}{ Salarios $(\Delta w(-3))$} & $-0.1649 * * *$ & 0.02070 & $-0.1580 * * *$ & 0.0181 \\
\hline & $(0.0203)$ & $(0.0366)$ & $(0.0292)$ & $(0.0391)$ \\
\hline \multirow[t]{2}{*}{ Salarios $(\Delta w(-4))$} & $0.5751 * * *$ & $0.7168 * * *$ & $0.6039 * * *$ & $0.7386 * * *$ \\
\hline & $(0.0451)$ & $(0.0465)$ & $(0.0601)$ & $(0.0574)$ \\
\hline \multirow{2}{*}{$\begin{array}{l}\text { Productividad } \\
(\Delta \varphi)\end{array}$} & -0.0215 & $0.0287 * *$ & -0.0142 & 0.02503 \\
\hline & $(0.0230)$ & $(0.0144)$ & $(0.0290)$ & $(0.0156)$ \\
\hline \multirow[t]{2}{*}{ Desempleo $(\Delta U n)$} & $-0.0253 *$ & -0.0188 & -0.0263 & -0.0205 \\
\hline & $(0.0149)$ & $(0.0127)$ & $(0.0179)$ & (0.01917) \\
\hline \multirow[t]{2}{*}{ Inflación $(\Delta \pi)$} & $0.0097 * * *$ & $0.0084 * * *$ & $0.0087 * *$ & $0.0067 *$ \\
\hline & $(0.0028)$ & $(0.0028)$ & $(0.0034)$ & $(0.0038)$ \\
\hline \multirow[t]{2}{*}{$\begin{array}{l}\text { Salario Mínimo } \\
(\triangle S M)\end{array}$} & 0.1271 & $0.0695 * *$ & 0.1405 & $0.0741 * * *$ \\
\hline & $(0.0823)$ & $(0.0229)$ & $(0.0854)$ & $(0.0275)$ \\
\hline Entidades & 32 & 32 & 32 & 32 \\
\hline Observaciones & 856 & 888 & 856 & 888 \\
\hline \multicolumn{5}{|l|}{ Prueba de } \\
\hline \multicolumn{5}{|l|}{$\begin{array}{l}\text { Autocorrelación } \\
\text { Arellano-Bond }\end{array}$} \\
\hline & \\
\hline \multicolumn{5}{|l|}{ Prueba de } \\
\hline Autocorrelación & {$[0.0775]$} & {$[0.9520]$} & {$[0.3285]$} & \\
\hline \multicolumn{5}{|l|}{ Arellano-Bond } \\
\hline \multicolumn{5}{|l|}{$\mathrm{AR}(1) \mathrm{a} /$} \\
\hline Prueba de Sargan b/ & {$[0.0000]$} & {$[0.0000]$} & [1.0000] & [1.0000] \\
\hline Prueba de Wald c/ & {$[0.0000]$} & {$[0.0000]$} & {$[0.0000]$} & {$[0.0000]$} \\
\hline
\end{tabular}

Fuente: Elaboración propia. Notas: Los errores estándar de los coeficientes se muestran entre paréntesis. Todas las variables son expresadas en primeras diferencias. Los $p$-value de las pruebas se muestran entre corchetes. *Coeficiente significativo al $10 \%$; **coeficiente significativo al 5\%; ***coeficiente significativo al $1 \%$. a/ $\mathrm{H}_{0}$ : no existe autocorrelación. b/ $\mathrm{H}_{0}$ : los instrumentos están bien definidos. c/ $\mathrm{H}_{0}$ : no existe heterocedasticidad. 
De dichos resultados, los signos para cada una de las variables contemporáneas corresponden, en su mayoría, a lo estimado a través del modelo estático, incluso variando el método de estimación. Para el caso de la variable dependiente con diferentes rezagos, resalta que considerando un retraso de 4 periodos, equivalente a un año, todos los coeficientes son positivos y significativamente diferentes de cero. Esto último sucede igualmente para el caso de la inflación; caso contrario, para la productividad, el salario mínimo y el desempleo, los niveles de significancia son limitados. Los resultados más robustos son aquellos que se obtienen de los modelos MGM de dos etapas. En ambos casos, las pruebas de Arellano y Bond confirman la presencia de correlación serial, considerando un proceso $\operatorname{AR}(1)$, pero no para un proceso AR(2). Igualmente, con la prueba de Sargan, se valida la correcta definición de los instrumentos elegidos. Con esto, los estimadores MGM2-DIFF y MGM2-SYS parecen ser los menos sesgados.

Considerando que estos resultados muestran diferencias principalmente entre los estimadores de una y dos etapas, es importante mencionar que Arellano y Bond (1991) señalan que los estimadores MGM2 presentan un sesgo a la baja, siendo sus errores estándar asintóticos, en promedio 30 por ciento menores a los asociados con las estimaciones de una etapa. Al respecto, en este caso, la corroboración de los resultados mediante dos estimadores diferentes valida la consistencia de los resultados. Dicho esto, el análisis se basará atendiendo a las últimas columnas $c$ y $d$ del cuadro 10, debido a que en estos casos se probó la correcta definición de sus instrumentos.

Referidos particularmente a la columna $c$, tratándose de un modelo cuya forma funcional establece primeras diferencias para variables logarítmicas, los resultados sugieren cambios relativos o porcentuales. En el caso de la variable dependiente rezagada a cuatro periodos, por cada unidad porcentual que estos cambien, se generarán incrementos en los salarios contemporáneos en aproximadamente 60 puntos base. Igualmente, la inflación tiene un impacto directo aunque a un nivel reducido, como sucedió en la estimación de la ecuación de salarios para el caso estático, donde por cada unidad porcentual que se incrementen los precios, los salarios (expresados en base logarítmica) se elevarán en aproximadamente un punto base.

En lo que corresponde a la estimación de la relación de largo plazo entre las variables para datos nuevamente mensuales, se excluye a la variable salarios mínimos debido a su poca significancia en los dos modelos anteriores, así como a la inflación, ya que para este caso no resulta posible evaluar ${ }^{3}$.

\footnotetext{
${ }^{3}$ Debido a que esta variable considera valores negativos, su transformación en logaritmo genera valores ausentes que impiden aplicar las pruebas de raíz unitaria.
} 
El cuadro 11 muestra los resultados correspondientes a las pruebas de raíz unitaria para cada una de las tres variables mencionadas, considerando, asimismo, los procesos autorregresivos que definen heterogeneidad entre las diferentes entidades. Para la variable desempleo, tres de las cinco pruebas confirman su estacionariedad, mientras que para el salario y la productividad, solo dos pruebas las definen como estacionarias. En especial, a través de las pruebas ADF-Fisher y PP-Fisher, podríamos nuevamente considerar a las tres variables como estacionarias, sin embargo, esto no resulta concluyente debido a los resultados de las otras pruebas.

\section{Cuadro 11}

Pruebas de Raíz Unitaria. Variables exógenas para efectos individuales y tendencia en el tiempo

\begin{tabular}{lccccrr}
\hline Var. & Breitung & $\begin{array}{c}\text { Levin, Lin } \\
\text { Chu }\end{array}$ & Hadri & $\begin{array}{c}\text { Im, Pesaran } \\
\text { and Shin }\end{array}$ & $\begin{array}{c}\text { ADF-Fisher } \\
(\text { Maddala y } \\
\text { Wu })\end{array}$ & $\begin{array}{c}\text { PP-Fisher } \\
\text { (Choi) }\end{array}$ \\
\hline $\ln w$ & 1.45 & 44.03 & $11.52 * *$ & 0.9 & $100.08 * *$ & $1520.74 * *$ \\
$\ln \varphi$ & $-5.04 * *$ & $-12.32 * *$ & $9.96 * *$ & $-14.31 * *$ & $401.23 * *$ & $634.2 * *$ \\
$\ln U n$ & 0.69 & $-3.89 * *$ & $23.4 * *$ & -0.82 & $85.8 * *$ & $87.92 * *$ \\
$\Delta \ln w$ & 0.68 & 163.3 & $4.26 * *$ & $-35.89 * *$ & $613.65 * *$ & $700.57 * *$ \\
$\Delta \ln \varphi$ & $-12.64 * *$ & $-49.75 * *$ & -4.08 & $-55.92 * *$ & $1599 * *$ & $1284.73 * *$ \\
$\Delta \ln U n$ & $-19.9 * *$ & $-32.15 * *$ & $2.96 * *$ & $-39.99 * *$ & $1202.9 * *$ & $1846.55 * *$ \\
\hline
\end{tabular}

Fuente: Elaboración propia. Notas: Las pruebas asumen una distribución asintótica normal, excepto las probabilidades para las pruebas de Fisher que usan una distribución asintótica Chi-cuadrada. *Indica el rechazo de la hipótesis nula de no estacionariedad (Breitung; Levin, Lin y Chu; Im, Pesaran y Shin; Maddala y Wu; y Choi) o estacionariedad (Hadri) para un nivel de significancia de al menos 5\%. $w$ : salarios, $\varphi$ : productividad, $U n$ : desempleo.

Ante lo anterior, es necesario recordar que las pruebas corresponden a escenarios diferentes. Las pruebas de Breitung, Levin, Li y Chu, y Hadri podrían resultar cuestionables si se considera el supuesto de convergencia absoluta, definido por un proceso homogéneo para todas las unidades de sección cruzada del panel. Desde luego, esto resulta complicado de mantener empíricamente, ya que, como se vio anteriormente, cada estado mantiene procesos diferentes en su estructura manufacturera, que dependen de la rama en que se especialicen así como de su tamaño relativo con respecto a otras actividades. Dicho esto, y tomando en cuenta principalmente las pruebas que rompen con este supuesto, es posible rechazar la hipótesis nula de no estacionariedad, lo cual permite continuar con la evaluación de la cointegración de largo plazo entre estas variables.

Para determinar la existencia de una relación de cointegración, el cuadro 12 muestra los resultados de las pruebas de cointegración de Pedroni y Kao. En él, se muestran dos grupos de estadísticos, cada uno sujeto a la hipótesis nula 
de no cointegración contra la hipótesis alternativa de cointegración. El primer grupo, denominado como estadísticas de panel, refiere a dimensiones "dentro de los grupos", asumiendo que existe un mismo término autorregresivo entre las secciones cruzadas; mientras que el segundo refiere a dimensiones "entre los grupos", donde el término varía para cada sección cruzada.

Cuadro 12

Pruebas de cointegración de panel. Salarios, productividad y desempleo, 2007-2015

\begin{tabular}{cllll}
\hline Prueba & \multicolumn{2}{c}{ Intercepto individual } & $\begin{array}{l}\text { Intercepto } \\
\text { individual }\end{array}$ & tendencia \\
\hline Cointegración residual de Pedroni & Estadístico & Probabilidad & Estadístico & Probabilidad \\
Panel estadístico v & 9.7294 & 0.0000 & 5.0107 & 0.0000 \\
Panel estadístico rho & -64.7280 & 0.0000 & -58.6974 & 0.0000 \\
Panel estadístico PP & -38.5165 & 0.0000 & -45.1962 & 0.0000 \\
Panel estadístico ADF & -9.9942 & 0.0000 & -11.5479 & 0.0000 \\
Grupo estadístico rho & -68.9005 & 0.0000 & -57.9958 & 0.0000 \\
Grupo estadístico PP & -48.1759 & 0.0000 & -49.9021 & 0.0000 \\
Grupo estadístico ADF & 1.9438 & 0.9740 & 3.3583 & 0.9996 \\
Cointegración residual de Kao & & & & \\
Estadístico ADF & 8.6287 & 0.0000 & - & - \\
\hline
\end{tabular}

Fuente: Elaboración propia. Notas: $\mathrm{H}_{0}$ : No cointegración.

Nuevamente, las pruebas de cointegración requieren de la especificación adecuada en cuanto a la forma funcional. En este caso, se eligió evaluar la regresión considerando un intercepto individual por cada sección cruzada, así como la regresión con intercepto y también con tendencia temporal individual. El número de rezagos fue determinado por el criterio de información de Shwarz (SIC), y la estimación fue hecha siguiendo el método de Bartlett.

En el caso de las estadísticas de panel, siguiendo el test de Pedroni, se rechaza la hipótesis nula en todos los casos para las tres variables incluidas en el modelo, lo cual indica cointegración. De la misma forma, en el caso de las estadísticas de grupo, únicamente la prueba ADF no permite rechazar la hipótesis nula. De manera conjunta, las pruebas de Pedroni ofrecen fuerte evidencia de una cointegración de panel entre las variables consideradas. La prueba de Kao corrobora el rechazo de la hipótesis nula de no cointegración entre las variables del modelo, con lo cual es posible definir una relación de largo plazo entre salarios, productividad y desempleo para la industria manufacturera mexicana. 
Una vez confirmada la combinación lineal de las series en el largo plazo, finalmente se procede a estimar el vector de cointegración. Para generar los estimadores en un panel de variables cointegradas, evitando los problemas de endogeneidad y autocorrelación que este tipo de información puede presentar, se recurre a los modelos FMOLS y DOLS, con los cuales efectivamente resulta posible definir estimadores consistentes. Para ambos casos, se consideran dos especificaciones: una con tendencia temporal lineal y otra con tendencia temporal constante. Asimismo, el número de rezagos para el modelo FMOLS, y de rezagos y adelantos para el modelo DOLS, fueron determinados a través del criterio de Schwarz.

De acuerdo con el cuadro 13, los resultados en cada uno de los modelos muestran coeficientes negativos para ambas variables y son estadísticamente significativos. Esto se entiende como la existencia de elasticidades con impacto inverso hacia los salarios por parte de la productividad y el desempleo. En el primer caso, el modelo FMOLS que considera una tendencia en el tiempo constante, la elasticidad de los salarios ante la productividad en el largo plazo es mayor a la elasticidad de los salarios ante el desempleo, aproximadamente -0.09 y -0.02 , respectivamente. Este resultado implica que, además de comprobar la relación inversa entre las variables, la magnitud coincide con los resultados obtenidos en el modelo estático, lo cual implica que la tendencia en el corto plazo se mantiene también en el largo plazo y con un mayor impacto de la productividad con respecto a la del desempleo.

\section{Cuadro 13}

Elasticidades de largo plazo para el panel cointegrado

\begin{tabular}{lll}
\hline Método & $\begin{array}{l}\text { Productividad } \\
(\ln \varphi)\end{array}$ & $\begin{array}{l}\text { Desempleo } \\
(\ln U n)\end{array}$ \\
\hline Fully Modified OLS $($ FMOLS $)$ & $-0.0877^{* * *}$ & $-0.0232^{*}$ \\
Con tendencia constante & $(0.0167)$ & $(0.0135)$ \\
Con tendencia lineal & $-0.0527^{* * *}$ & $-0.0453^{* * *}$ \\
& $(0.0142)$ & $(0.0119)$ \\
& $-0.0628^{* * *}$ & $-0.0250^{*}$ \\
Dynamic OLS (DOLS) & $(0.0186)$ & $(0.0138)$ \\
Con tendencia constante & $-0.0258^{*}$ & $-0.0366^{* * *}$ \\
Con tendencia lineal & $(0.0158)$ & $(0.0120)$ \\
\hline
\end{tabular}

Fuente: Elaboración propia. Notas: Los errores estándar de los coeficientes se muestran en paréntesis. Todas las variables son expresadas en logaritmos. *Coeficiente significativo al $10 \%$; **coeficiente significativo al $5 \%$; ***coeficiente significativo al $1 \%$. 
Ahora bien, considerando una tendencia lineal en el tiempo, lo cual es más adecuado para explicar la dependencia entre las secciones transversales, las elasticidades de largo plazo varían ligeramente a la baja con respecto a los primeros resultados sin tendencia, siendo estos de -0.05 y -0.04 , en cada caso. Igualmente, este patrón se confirma con el cálculo de los estimadores mediante DOLS, aunque en niveles inferiores en todos los casos, pero siempre manteniéndose de manera cercana.

Como síntesis, el cuadro 14 muestra los coeficientes estimados para las variables de la ecuación de salarios propuesta en cada uno de los modelos evaluados en este apartado. Cabe aclarar que en todos los casos, esta síntesis de resultados refiere al total del conjunto de datos, por lo que no integra las agrupaciones por tiempo y estados para el caso del modelo estático.

Cuadro 14

\section{Síntesis de resultados. Variable dependiente: salarios (w)}

\begin{tabular}{|c|c|c|c|c|c|}
\hline Modelo & $\begin{array}{c}\text { Salarios } \\
(w(-4))\end{array}$ & $\begin{array}{l}\text { Productivid } \\
\quad \operatorname{ad}(\varphi)\end{array}$ & $\begin{array}{l}\text { Desempleo } \\
\text { (Un) }\end{array}$ & $\begin{array}{c}\text { Inflación } \\
(\pi)\end{array}$ & $\begin{array}{c}\text { Salario } \\
\text { Mínimo } \\
(S M)\end{array}$ \\
\hline \multicolumn{6}{|c|}{ Modelo estático a/ } \\
\hline MCO & - & $-0.0787 * * *$ & $-0.0371 * * *$ & $0.0093 * * *$ & 0.0106 \\
\hline MEF1 & - & $-0.0787 * * *$ & $-0.0371 * * *$ & $0.0093 * * *$ & 0.0106 \\
\hline MEA & - & $-0.0640 * * *$ & $-0.0370 * * *$ & $0.0093 * * *$ & 0.0068 \\
\hline MEF2 & - & $-0.0787 * *$ & $-0.0371^{*}$ & $0.0093 * * *$ & 0.0106 \\
\hline MCP & - & $0.2177 * * *$ & $-0.0236^{* *}$ & 0.0061 & $-0.0868 * *$ \\
\hline \multicolumn{6}{|c|}{ Modelo dinámico b/ } \\
\hline MGM1-DIFF & $0.5751 * * *$ & -0.0215 & $-0.0253^{*}$ & $0.0097 * * *$ & 0.1271 \\
\hline MGM1-SYS & $0.7168 * * *$ & $0.0287 * *$ & -0.0188 & $0.0084 * * *$ & $0.0695^{* *}$ \\
\hline MGM2-DIFF & $0.6039 * * *$ & -0.0142 & -0.0263 & $0.0087 * *$ & 0.1405 \\
\hline MGM2-SYS & $0.7386^{* * *}$ & 0.025 & -0.0205 & $0.0067^{*}$ & $0.0741 * * *$ \\
\hline \multicolumn{6}{|c|}{ Modelo de largo plazo a/ } \\
\hline FMOLS1 & - & $-0.0877 * * *$ & $-0.0232 *$ & - & - \\
\hline FMOLS2 & - & $-0.0527 * * *$ & $-0.0453 * * *$ & - & - \\
\hline DOLS1 & - & $-0.0628 * * *$ & $-0.0250^{*}$ & - & - \\
\hline DOLS2 & - & $-0.0258^{*}$ & $-0.0366^{* * *}$ & - & - \\
\hline
\end{tabular}

Fuente: Elaboración propia. Notas: *Coeficiente significativo al 10\%; **coeficiente significativo al 5\%; ***coeficiente significativo al 1\%. FMOLS1: Modelo FMOLS con tendencia constante; FMOLS2: Modelo FMOLS con tendencia lineal; DOLS1: Modelo DOLS con tendencia constante; DOLS2: Modelo DOLS con tendencia lineal. a/ Modelo con variables logarítmicas; b/ Modelo con variables en primeras diferencias. 


\section{Conclusiones}

Los resultados permitieron corroborar que los salarios tienen una respuesta ante variaciones, tanto en la productividad laboral como en el desempleo. Sin embargo, dichas relaciones tienen signo inverso al esperado en el caso de la productividad laboral, mientras que para el caso del desempleo, el signo negativo concuerda con la teoría económica y los estudios empíricos, aunque en un nivel reducido pero significativo. Los resultados sugieren una elasticidad salarios-desempleo cercana al -0.04 , la cual se aproxima a los resultados de la curva de salarios de Castro (2006).

La reducción de los salarios debido a incrementos en la productividad diverge tanto de los planteamientos teóricos neoclásicos y neokeynesianos, como de la evidencia empírica reciente. Se presume que este resultado podría tener fundamento, dado el bajo costo de la mano de obra mexicana, en los mecanismos de atracción de IED. Dicho de otra forma, al considerar el actual contexto internacional caracterizado por una mayor competencia entre países de mano de obra de bajo costo y poco especializada, la contención de los salarios resulta importante para continuar con la atracción de capital extranjero. Retomando el caso de Malasia, analizado por Goh y Wong (2010), los altos impactos directos transmitidos de la productividad laboral hacia los salarios explican las disminuciones en la IED.

Resulta prudente sugerir para estudios posteriores, el análisis de variables que aproximen el comportamiento de IED para evaluar su impacto hacia los salarios y la productividad laboral. La información disponible al momento en fuentes oficiales impide la integración de una variable de este tipo, principalmente tratándose de datos en el nivel estatal y con periodicidad mensual.

En línea con lo anterior, el uso de un panel de datos que incluye información de 32 entidades con comportamientos particulares, así como el empleo de técnicas econométricas estáticas, dinámicas y de largo plazo que convergen en los resultados, da consistencia a la evidencia encontrada. Más aún, este patrón inverso entre productividad y salarios se observa principalmente en entidades cuya actividad económica cuenta con una mayor participación por parte de la industria manufacturera, en términos de empleo y producción; como ejemplo están los casos de: Estado de México, Nuevo León, Coahuila, Chihuahua, Guanajuato y la Ciudad de México y, únicamente Jalisco, como excepción.

Con respecto al impacto por parte de la inflación y el salario mínimo, se encontró que únicamente en el primer caso existe una relación positiva y significativa, aun cuando se incorpora el factor dinámico dentro de la 
ecuación de salarios. Lo anterior corresponde igualmente con lo esperado, toda vez que el salario debe absorber los incrementos en los precios para mantener su poder adquisitivo. Sin embargo, aunque resulta positivo y significativo, el coeficiente para esta variable es reducido (0.009). Por su parte, el salario mínimo no mostró efectos, que se mantuvieran de manera consistente, en cada uno de los modelos estimados.

En conjunto, el comportamiento descrito para cada una de las variables consideradas supone que los salarios en México son relativamente rígidos, de manera tal que en el corto plazo, o aun en el largo plazo, no ejercen un ajuste significativo ante cambios en las condiciones del mercado.

Por otra parte, los salarios rezagados un año resultan relevantes para explicar los salarios actuales. En este caso, el coeficiente obtenido es positivo y oscila alrededor del 0.65 ; lo cual, como se observa, es mayor a los efectos generados a partir de la productividad o el desempleo. En efecto, estas variables pierden significancia una vez que se considera el comportamiento pasado de los salarios. Este resultado tiene correspondencia con la periodicidad de las negociaciones salariales entre empleadores y trabajadores, lo cual está vinculado al entorno institucional de los contratos laborales y los sindicatos. De esta manera, las variaciones en los salarios generadas a partir de elementos económicos del mercado laboral podrían más bien tener una importancia secundaria.

En síntesis, considerando el reducido impacto y crecimiento de la productividad laboral, la insuficiente capacidad de los salarios para absorber las variaciones de la inflación y los cambios en el salario mínimo que no resultan significativos, los resultados permiten explicar el estancamiento por el que atraviesan los salarios en México. Debido a que los salarios son una de las variables más importantes en la economía, cuando se trata del ingreso de la población, las variables económicas resultan insuficientes para incidir de forma importante sobre los salarios. Por el contrario, en el caso mexicano, se puede establecer que una de las variables económicas más importantes no se explica a partir de condiciones de la misma economía doméstica; sino que, más bien, el crecimiento significativo de los salarios puede surgir tanto de transformaciones institucionales como de los cambios de la economía interna e internacional.

\section{Referencias}

[1] Adams, J. D. (1985). "Permanent Differences in Unemployment and Permanent Wage Differentials". The Quarterly Journal of Economics, 100(1), 29-56.

[2] Akerlof, G. A. (1980). "A Theory of Social Custom, of Which Unemployment May Be One Consequence”. The Quarterly Journal of Economics, 94(4), 749775 . 
[3] Akerlof, G. A. y Yellen, J. L. (1990). "The Fair Wage-Effort Hypothesis and Unemployment”. The Quarterly Journal of Economics, Mayo, 105(2), 255283.

[4] Alexander, C. O. (1993). "The Changing Relationship Between Productivity, Wages and Unemployment in the UK". Oxford Bulletin of Economics and Statistics, 55(1), 877-102.

[5] Álvarez, J. y Arellano, M. (2003). "The Time Series and Cross-Section Asymptotics of Dynamic Panel Data Estimators”. Econometrica, Julio, 71(4), 1121-1159.

[6] Arellano, M. y Bond, S. (1991). "Some Tests of Specification for Panel Data: Monte Carlo Evidence and an Application to Employment Equations". The Review of Economic Studies, Abril, 58(2), 277-297.

[7] Azariadis, C. y Stiglitz, J. E. (1983). "Implicit Contracts and Fixed Price Equilibria”. The Quarterly Journal of Economics, 98, supplement, 1-22.

[8] Baltagi, B. H. (2005). Econometric Analysis of Data Panel. 3a ed. Inglaterra: John Wiley y Sons Ltd.

[9] Blackaby, D. H. y Manning, D. N. (1987). "Regional Earnings Revisited". The Manchester School, Junio, 55(2), 158-183.

[10] Blanchard, O. J. y Katz, L. F. (1992). "Regional Evolutions". Brookings Papers on Economic Activity, 1, 1-75.

[11] Blanchard, O. J. y Summers, L. H. (1987). "Hysteresis in Unemployment". European Economic Review, 31(1/2), 288-295.

[12] Blanchflower, D. G. y Oswald, A. J. (1990). "The Wage Curve". The Scandinavian Journal of Economics, 2(92), 215-235.

[13] Blanchflower, D. G. y Oswald, A. J. (1994). "Estimating a Wage Curve for Britain 1973-1990”. The Economic Journal, 104, 1025-1043.

[14] Breitung, J., (2001). "The Local Power of Some Unit Root Tests for Panel Data". En Baltagi, B. H., Fomby T. B. y Carter Hill R. (Eds), Nonstationary Panels, Panel Cointegration, and Dynamic Panels. s.l. (161-177), Emerald Group Publishing Limited.

[15] Castellanos, S. G. (2010). "Desempleo y Determinacion de Salarios en la Industria Manufacturera de México". Economía Mexicana, Enero-Junio, XIX(1), 171-198.

[16] Castro, D. (2006). "Curva salarial: una aplicación para el caso de México, 19932002”. Estudios Económicos, Julio-Diciembre, 21(2), 233-273.

[17] CEFP. (2004). "Evolucion del Sector Manufacturero de México, 1980-2003". Centro de Estudios de las Finanzas Públicas, Cámara de de Diputados, México.

[18] Choi, I. (2001). "Unit Root Tests for Panel Data”. 20(1), 249-272.

[19] Engle, R. F. y W.J. Granger, W, J. (1987). "Cointegration and error correction: Representation,

[20] estimation and testing”. Econometrica, 55, 251-276.

[21] Freeman, R. B. (1988). "Evaluating the European View that the United States Has No Unemployment Problem”. The American Economic Review, Mayo, 78(2), 294-299.

[22] Friedman, M. (1968). "The Role of Monetary Policy". American Economic Review, Marzo, LVIII(1), 1-17.

[23] Galindo, L. M. y Catalán, H. (2010). “El Mercado Laboral en México: ¿Una Curva de Salarios?". Comercio Exterior, Marzo, 60(3), 221-232. 
[24] Goh, S. K. y Wong, K. N. (2010). "Analyzing the Productivity-WageUnemployment Nexus in Malaysia: Evidence from the Macroeconomic Perspective". International Research Journal of Finance and Economics, Volume 53, 145-156.

[25] Gregory, R. G. (1986). "Wages Policy and Unemployment in Australia". Economica, New Series, 53(210, supplement), S53-S74.

[26] Hadri, K. (2000). "Testing for Stationarity in Heterogeneus Panel Data". Econometric Journal, 3(1), 148-161.

[27] Hall, R. E., Gordon, A. y Holt, C. (1972). "Turnover in the Labor Force". Brookings Papers on Economic Activity, 1972(3), 709-764.

[28] Harris, J. R. y Todaro, M. P. (1970). "Migration, Unemployment and Development: A Two-Sector Analysis". American Economic Review, 60(1), 126-142.

[29] Im, K. S., Pesaran, M. H. y Shin, Y. (2003). "Testing for Unit Roots in Heterogeneous Panels". Journal of Economics, Julio, 115(1), 53-74.

[30] INEGI (2016). Instituto Nacional de Estadística y Geografía (INEGI). [Online] Disponible en: www.inegi.org.mx [Acceso 09 Mayo 2016].

[31] Jones, S. R. G. (1989). "Reservation Wages and the Cost of Unemployment". Economica, Mayo, 56(222), 225-246.

[32] Kao, C. y Chiang, M. H. (2001). "On the Estimation and Inference of a Cointegrated Regression in Panel Data". En: Baltagi, B. H., Fomby T.B. y Hill R.C. (Eds). Nonstationary Panels, Panel Cointegration, and Dynamic Panels. s.1.(179-222):Emerald Group Publishing Limited.

[33] Kumar, S., Webber, D. J. y Perry, G. (2012). "Real wages, inflation and labour productivity in Australia”. Applied Economics, 44(23), 2945-2954.

[34] Lavy, J. y Sussman, N. (2001). The Determination of Real Wages in the Long Run and its Changes in the Short Run: Evidence from Israel, s.l.: Research Department, Bank of Israel.

[35] Layard, R. y Nickell, S. (1986). "Unemployment in Britain". Economica, 53(210), S121-S169.

[36] Levin, A., Lin, C. F. y Chu, C.-S. J. (2002). "Unit Root Tests in Panel Data: Asymptotic and Finite-Sample Properties". Journal of Econometrics, 108(1), $1-24$.

[37] Lindbeck, A. y Snower, D. J. (1986). "Wage Setting, Unemployment, and Insider-Outsider Relations". The American Economic Review, Mayo, 76(2), 235-239.

[38] Maddala, G. S. y Wu, S. (1999). “A Comparative Study of Unit Root Tests with Panel Data and a New Simple Test”. Oxford Bulletin of Economics and Statistics, Noviembre, 61(1), 631-652.

[39] Marston, S. T. (1985). "Two Views of the Geographic Distribution of Unemployment". The Quarterly Journal of Economics , 100(1), 57-79.

[40] Mendoza, E. y Martínez , G. (1999). “Globalización y Dinámica Industrial en la Frontera Norte de México”. Comercio Exterior, 49(9), 795-806.

[41] Mortensen, D. T. (1986). "Job Search and Labor Market Analysis". En: Ashenfelter, O.y Layard, R. (Eds). Handbook of Labor Economics. Amsterdam(North-Holland): s.n., 849-919.

[42] Pedroni, P. (1999). "Critical Values for Cointegration Tests in Heterogeneous Panels with Multiple Regressors". Oxford Bulletin of Economics and Statistics, Noviembre, 61( 1), 653-670. 
[43] Phelps, E. S. (1968). "Money-Wage Dynamics and Labor-Market Equilibrium". Journal of Political Economy, 76( 4), 679-711.

[44] Phillips, A. W. (1958). "The Relation Between Unemployment and the Rate of Change of Money Wage Rates in the United Kingdom, 1861-1957'. Economica, 25(100), 283-299.

[45] Phillips, P. C. B. y Hansen, B. E. (1990). "Statistical Inference in Instrumental Variables Regression with I(1) Processes". The Review of Economic Studies , 57( 1), 99-125.

[46] Pissarides, C. A. y MacMaster, I. (1990). "Regional Migration, Wages and Unemployment: Empirical Evidence and Implications for Policy". Oxford Economic Papers, 42(4), 812-831.

[47] Reza, A. M. (1978). "Geographical Differences in Earnings and Unemployment Rates”. The Review of Economics and Statistics, 60( 2), 201-208.

[48] Rivera, M. Á. (2001). "México en la Economía Global: Reinserción Aprendizaje y Coordinación". Problemas de Desarrollo, 32(127), 75-105.

[49] Roback, J. (1982). "Wages, Rents, and the Quality of Life". Journal of Political Economy, 90( 6), 1257-1278.

[50] Saikkonen, P. (1992). "Estimation and Testing of Cointegrated Systems by an Autoregressive Approximation". Econometric Theory, 8( 1), 1-27.

[51] Shapiro, C. y Stiglitz, J. E. (1984). "Equilibrium Unemployment as a Worker Discipline Device". American Economic Association, 74( 3), 433-444.

[52] Stiglitz, J. E. (1986). “Theories of Wage Rigidity”. En Butkiewicz, J. L., Koford, K. J. y Miller, J. B. (Eds). Keynes' Economic Legacy: Contemporary Economic theories. New York: Praeger Publishers, 153-206.

[53] Stock, J. y Watson, M. W. (1993). “A Simple Estimator of Cointegrating Vectors in Higher-Order Integrated Systems". Econometrica, 61(4), 783-820.

[54] Strauss, J. y Wohar, M. E. (2004). "The Linkage between Prices, Wages, and Labor Productivity: A Panel Study of Manufacturing”. Southern Economic Journal, 70( 4), 920-941.

[55] Topel, R. H. (1986). "Local Labor Markets". 94, 3, S111-S143.

[56] Wakeford, J. (2004). "The Productivity-Wage Relationship in South Africa: An Empirical Investigation". Development Southern Africa, 21( 1), 109-132.

[57] Wanger, M. y Hlouskova, J. (2007). "The Performance of Panel Cointegration Methods: Results from a Large Scale Simulation Study". Economics Series, $1-45$. 